\title{
K3 SURFACES WITH NON-SYMPLECTIC AUTOMORPHISMS OF PRIME ORDER
}

\author{
MICHELA ARTEBANI, ALESSANDRA SARTI, SHINGO TAKI
}

(WITH AN APPENDIX BY SHIGEYUKI KONDŌ)

\begin{abstract}
In this paper we present the classification of non-symplectic automorphisms of prime order on $K 3$ surfaces, i.e. we describe the topological structure of their fixed locus and determine their invariant lattice in cohomology. We provide new results for automorphisms of order 5 and 7 and alternative proofs for higher orders. Moreover, for any prime $p$, we identify the irreducible components of the moduli space of $K 3$ surfaces with a non-symplectic automorphism of order $p$.
\end{abstract}

\section{INTRODUCTION}

A $K 3$ surface is a compact surface $X$ over $\mathbb{C}$ with trivial canonical bundle and $\operatorname{dim} H^{1}\left(X, \mathcal{O}_{X}\right)=0$. In the following, we will denote by $S_{X}, T_{X}$ and $\omega_{X}$ the Picard lattice, the transcendental lattice and a nowhere vanishing holomorphic 2 -form on $X$, respectively.

An automorphism $\sigma$ of a $K 3$ surface $X$ is called symplectic if it acts trivially on $\mathbb{C} \omega_{X}$. This paper deals with $K 3$ surfaces carrying a non-symplectic automorphism of prime order $p$. In this case it is known that $\sigma$ acts without non zero fixed vectors on the transcendental lattice, so that $T_{X}$ acquires the structure of a module over $\mathbb{Z}\left[\zeta_{p}\right]$, where $\zeta_{p}=e^{2 \pi i / p}$. Since $T_{X}$ is contained in the rank 22 lattice $H^{2}(X, \mathbb{Z})$, this implies that $p$ is at most 19 .

Non-symplectic automorphisms have been studied by several authors, e.g. in 32, 20, 21, 2, 31, 22, 23, 24, 14. It is known that for $p=13,17,19$ there are only isolated pairs $\left(X_{p}, \sigma_{p}\right)$, where $\sigma_{p}$ is a non-symplectic automorphism of order $p$ acting on the $K 3$ surface $X_{p}$. This was first announced (without proof) by Vorontsov in [32] and then it was proved by Kondō in [11] and Oguiso-Zhang in [23].

In this paper we give the classification for $p=5,7$, we survey the known results for $p=2,3$ and we provide different proofs and examples for $p=11,13,17,19$. The key idea is to characterize the fixed locus of the automorphism in terms of the properties of its invariant lattice in $H^{2}(X, \mathbb{Z})$. The main result is the following.

Theorem 0.1. Let $S$ be a hyperbolic p-elementary lattice ( $p$ prime) of rank $r$ with $\operatorname{det}(S)=p^{a}$. Then $S$ is isometric to the invariant lattice of a non-symplectic automorphism $\sigma$ of order $p$ on a K3 surface if and only if

$$
\text { (*) } \quad 22-r-(p-1) a \in 2(p-1) \mathbb{Z}_{\geq 0} .
$$

2000 Mathematics Subject Classification. Primary 14J28; Secondary 14J50, 14J10.

Key words and phrases. K3 surface, non-symplectic automorphism, lattice.

The first author has been partially supported by Proyecto FONDECYT Regular 2009, N. 1090069. 
Moreover, if $\sigma$ is such automorphism, then its fixed locus $X^{\sigma}$ is the disjoint union of smooth curves and isolated points and has the following form:

$$
X^{\sigma}=\left\{\begin{array}{lc}
\emptyset & \text { if } S \cong U(2) \oplus E_{8}(2), \\
E_{1} \cup E_{2} & \text { if } S \cong U \oplus E_{8}(2), \\
C \cup R_{1} \cup \cdots \cup R_{k} \cup\left\{p_{1}, \ldots, p_{n}\right\} & \text { otherwise, }
\end{array}\right.
$$

where $E_{i}$ is a smooth elliptic curve, $R_{i}$ is a smooth rational curve, $p_{i}$ is an isolated fixed point and $C$ is a smooth curve of genus

$$
g=\frac{22-r-(p-1) a}{2(p-1)} .
$$

Moreover:

\begin{tabular}{c|cccccc} 
& $p=2$ & $p=3,5,7$ & $p=11$ & $p=13$ & $p=17$ & $p=19$ \\
\hline$n$ & 0 & $\frac{-2+(p-2) r}{p-1}$ & $\frac{2+9 r}{10}$ & 9 & 7 & 5 \\
$k$ & $\frac{r-a}{2}$ & $\frac{2+r-(p-1) a}{2(p-1)}$ & $\frac{-2+r-10 a}{20}$ & 1 & 0 & 0
\end{tabular}

with the convention that $X^{\sigma}$ contains no fixed curves if $k=-1$.

As a consequence of this result, we determine the maximal components of the moduli space of $K 3$ surfaces with a non-symplectic automorphism of order $p$ for any prime $p$. Moreover, we show that for $p>2$ the topological structure of the fixed locus of $\sigma$ determines uniquely the action of $\sigma$ on $H^{2}(X, \mathbb{Z})$ (see Remark 2.10).

The plan of the paper is the following. Section 1 introduces some background material on lattices. In Section 2 we prove the main properties of the invariant lattice $S(\sigma)$ and of the fixed locus of a non-symplectic automorphism $\sigma$ of prime order. Theorem 2.4 gives the number of isolated fixed points and the local action at them, as a function of the rank of $S(\sigma)$. Moreover, by means of Smith exact sequences, we determine the maximal genus $g$ of a fixed curve as a function of the lattice invariants of $S(\sigma)$, see Corollary 2.9. These results show that the topology of the fixed locus of the automorphism is uniquely determined by the isometry class of its invariant lattice and allow to give a complete classification. The following sections give more explicit results for each prime order $p$.

Sections 3 and 4 briefly review the known results for $p=2$ and 3 respectively. All possible configurations of the fixed locus related to the invariants of $S(\sigma)$ are represented in Figures 1 and 2 .

In section 5 we classify non-symplectic automorphisms of order 5 . The classification theorem is resumed in Table 2. The topological structure of the fixed locus gives a natural stratification in 7 families. Two of them, of dimensions 3 and 4 , are the maximal irreducible components of the moduli space (as we will show in section 10). We provide projective models for the generic member of each family.

In section 6 we give a similar classification and description for $p=7$, see Table 3. In this case there are two maximal components of dimension 2. 
In sections 7 and 8 we provide an alternative view of the classification for $p=$ $11,13,17,19$. In case $p=11$ it is known that the moduli space has two maximal 1-dimensional components, while the pair $(X, \sigma)$ is unique for $p>11$.

In section 9 we deal with moduli spaces. First, we recall the structure of the moduli space of pairs $(X, \sigma)$ where $X$ is a $K 3$ surface and $\sigma$ is a non-symplectic automorphism of order $p$ with a given representation in $H^{2}(X, \mathbb{Z})$. This moduli space is known to be isomorphic to a quotient of an open dense subset of either an Hermitian symmetric domain of type $I V$ (for $p=2$ ) or of a complex ball (for $p>2$ ) for the action of a discrete group. For any prime $p$ we identify the irreducible components of the moduli space of $K 3$ surfaces with a non-symplectic automorphism of order $p$, see Theorem 9.5 .

In the appendix $\mathrm{S}$. Kondō shows that the moduli space of pairs $(X, \sigma)$, where $\sigma$ is non-symplectic of order 7 having only isolated fixed points, is a ball quotient isomorphic to the Naruki $K 3$ surface. A similar example for $p=5$ was given by the same author in [12.

Acknowledgements. We would like to thank Igor Dolgachev, Alice Garbagnati and the referee for several helpful comments.

\section{LATTICES}

A lattice is a finitely generated free abelian group equipped with a non-degenerate symmetric bilinear form with integer values. If the signature of the lattice is $(1, r-1)$ then it is called hyperbolic. We will work with even lattices i.e. such that the quadratic form on it takes values in $2 \mathbb{Z}$.

The quadratic form on $L$ determines a canonical embedding $L \subset L^{*}=\operatorname{Hom}(L, \mathbb{Z})$. We denote by $A_{L}$ the factor group $L^{*} / L$, which is a finite abelian group. If this group is trivial, then $L$ is called unimodular.

Let $p$ be a prime number. A lattice $L$ is called p-elementary if $A_{L} \simeq \mathbb{Z}_{p}^{a}$. If $L$ is a $p$-elementary lattice primitively embedded in a unimodular lattice $M$ and $L^{\perp}$ is its orthogonal complement in $M$, then it is known that $L^{\perp}$ is also $p$-elementary and $p^{a}=|\operatorname{det}(L)|=\left|\operatorname{det}\left(L^{\perp}\right)\right|$.

The following result classifies even, indefinite, $p$-elementary lattices (see [27]).

Theorem 1.1. An even, indefinite, $p$-elementary lattice of rank $r$ for $p \neq 2$ and $r \geq 2$ is uniquely determined by the integer $a$.

For $p \neq 2$ a hyperbolic $p$-elementary lattice with invariants a, $r$ exists if and only if the following conditions are satisfied: $a \leq r, r \equiv 0(\bmod 2)$ and

$$
\left\{\begin{array}{llll}
\text { for } a \equiv 0 & (\bmod 2), & r \equiv 2 \quad(\bmod 4) \\
\text { for } a \equiv 1 & (\bmod 2), & p \equiv(-1)^{r / 2-1} \quad(\bmod 4) .
\end{array}\right.
$$

Moreover $r>a>0$, if $r \not \equiv 2(\bmod 8)$.

An even, indefinite, 2-elementary lattice is determined by $r$, a and a third invariant $\delta \in\{0,1\}$, see $[20$.

Notation. We will denote by $U$ the unique even unimodular hyperbolic lattice of rank two and by $A_{m}, D_{n}, E_{l}$ the even, negative definite lattices associated with the Dynkin diagrams of the corresponding types $(m \geq 1, n \geq 4, l=6,7,8)$. Moreover, $L(a)$ and $L^{b}$ will denote the lattices whose bilinear form is respectively the one on $L$ multiplied by $a$ and the orthogonal sum of $b$ copies of the one on $L$. 


\section{Examples.}

- The lattices $U$ and $E_{8}$ are unimodular. Any even unimodular lattice of signature $(3,19)$ is isometric to $L_{K 3}=U^{\oplus 3} \oplus E_{8}^{\oplus 2}([15,28])$.

- If $p$ is prime, then the lattice $A_{p-1}$ is $p$-elementary with $a=1$.

- The lattice $E_{7}$ is 2-elementary with $a=1$.

- If $p \equiv 3(\bmod 4)$, then the lattice

$$
K_{p}=\left(\begin{array}{cc}
-(p+1) / 2 & 1 \\
1 & -2
\end{array}\right)
$$

is negative definite, $p$-elementary, with $a=1$. Note that $K_{3} \cong A_{2}$.

- If $p \equiv 1(\bmod 4)$ then the lattice

$$
H_{p}=\left(\begin{array}{cc}
(p-1) / 2 & 1 \\
1 & -2
\end{array}\right)
$$

is hyperbolic, $p$-elementary, with $a=1$.

- The lattice

$$
A_{4}^{*}(5)=\left(\begin{array}{rrrr}
-4 & 1 & 1 & 1 \\
1 & -4 & 1 & 1 \\
1 & 1 & -4 & 1 \\
1 & 1 & 1 & -4
\end{array}\right)
$$

is negative definite, 5 -elementary with $a=3$.

- The lattice

$$
L_{17}=\left(\begin{array}{rrrr}
-2 & 1 & 0 & 1 \\
1 & -2 & 0 & 0 \\
0 & 0 & -2 & 1 \\
1 & 0 & 1 & -4
\end{array}\right)
$$

is negative definite, 17-elementary with $a=1$.

\section{NON-SYMPLECTIC AUTOMORPHISMS ON $K 3$ 's}

Let $X$ be a $K 3$ surface i.e. a simply connected smooth compact complex surface with a nowhere vanishing holomorphic 2 -form $\omega_{X}$. The cohomology group $H^{2}(X, \mathbb{Z})$, equipped with the cup product, is known to be an unimodular lattice isometric to $L_{K 3}$. The Picard lattice $S_{X}$ and the transcendental lattice $T_{X}$ are the following primitive sublattices of $H^{2}(X, \mathbb{Z})$ :

$$
S_{X}=\left\{x \in H^{2}(X, \mathbb{Z}):\left(x, \omega_{X}\right)=0\right\}, \quad T_{X}=S_{X}^{\perp} .
$$

An automorphism $\sigma$ of $X$ is called non-symplectic if its action on the vector space $H^{2,0}(X)=\mathbb{C} \omega_{X}$ is not trivial. Observe that, by [18, Theorem 3.1], K3 surfaces with a non-symplectic automorphism of finite order are always algebraic. In this paper we are interested in non-symplectic automorphisms of prime order i.e.

$$
\sigma^{p}=i d \quad \text { and } \quad \sigma^{*}\left(\omega_{X}\right)=\zeta_{p}^{k} \omega_{X}, 0<k<p,
$$

where $\zeta_{p}$ is a primitive $p$-th root of unity.

The automorphism $\sigma$ induces an isometry $\sigma^{*}$ on $H^{2}(X, \mathbb{Z})$ which preserves both $S_{X}$ and $T_{X}$. We will consider the invariant lattice and its orthogonal in $H^{2}(X, \mathbb{Z})$ :

$$
S(\sigma)=\left\{x \in H^{2}(X, \mathbb{Z}): \sigma^{*}(x)=x\right\}, \quad T(\sigma)=S(\sigma)^{\perp} .
$$

Theorem 2.1. Let $X$ be a K3 surface and $\sigma$ be a non-symplectic automorphism of $X$ of prime order $p$. Then 
a) $S(\sigma) \subset S_{X}$ and $T_{X} \subset T(\sigma)$;

b) $T(\sigma)$ and $T_{X}$ are free modules over $\mathbb{Z}\left[\zeta_{p}\right]$ via the action of $\sigma^{*}$;

c) $S(\sigma)$ and $T(\sigma)$ are $p$-elementary lattices and $A_{S(\sigma)} \cong A_{T(\sigma)} \cong \mathbb{Z}_{p}^{a}$ with

$$
a \leq \frac{\operatorname{rank}(T(\sigma))}{p-1} .
$$

Proof. The statements $a$ ), b) and the first claim in $c$ ) are proved in [18, Section 3] or [14, Lemma 1.1]. For the inequality in $c$ ), we will generalize the proof of [14, Claim 3.4] as follows. By point $b$ ) we have that $T(\sigma) \cong \mathbb{Z}\left[\zeta_{p}\right]^{m}$ as a $\mathbb{Z}\left[\zeta_{p}\right]$-module. Let $e_{1}, \ldots, e_{m}$ be a basis of $T(\sigma)$ over $\mathbb{Z}\left[\zeta_{p}\right]$ and let

$$
\left\{b_{i j}: i=1, \ldots, m, j=0, \ldots, p-2\right\}
$$

be the corresponding $\mathbb{Z}$ basis of $T(\sigma)$. Since $T(\sigma)$ is $p$-elementary, then any $y \in$ $T(\sigma)^{*}$ is of the form

$$
y=\frac{1}{p} \sum_{i, j} y_{i j} b_{i j}, \quad y_{i j} \in \mathbb{Z} .
$$

Moreover, since $\sigma^{*}=i d$ on $S(\sigma)$, then $\sigma^{*}=i d$ on $A_{S(\sigma)} \cong A_{T(\sigma)}$. Thus modulo $T(\sigma)$ we have

$$
0 \equiv \sigma^{*}(y)-y=\frac{1}{p} \sum_{i=1}^{m}\left(\sum_{j=0}^{p-3} y_{i j} b_{i j+1}-y_{i p-2}\left(b_{i 0}+\cdots+b_{i p-2}\right)-\sum_{j=0}^{p-2} y_{i j} b_{i j}\right) .
$$

From the vanishing of the coefficients of the $b_{i j}$ 's it follows that $y_{i j} \equiv(j+1) y_{i 0}$ modulo $p$. Thus

$$
\frac{1}{p} \sum_{j=0}^{p-2} y_{i j} b_{i j} \equiv y_{i 0}\left[\frac{1}{p} \sum_{j=0}^{p-2}(j+1) b_{i j}\right]=y_{i 0} B_{i} .
$$

This implies that $A_{T(\sigma)}$ is generated by $B_{1}, \ldots, B_{m}$, hence $a \leq m$.

In what follows we will denote by $m=(22-r) /(p-1)$ the rank of $T(\sigma)$ as a $\mathbb{Z}\left[\zeta_{p}\right]$-module ( $r$ denotes the rank of $S(\sigma)$ ).

We will now describe the structure of the fixed locus $X^{\sigma}$ of a non-symplectic automorphism $\sigma$ of order $p$ of a $K 3$ surface. We can assume $\sigma$ to act on $\omega_{X}$ as the multiplication by $\zeta_{p}$. The action of $\sigma$ can be locally linearized and diagonalized at a fixed point $x \in X^{\sigma}$ (see $\S 5,[18$ ), so that its possible local actions are

$$
A_{p, t}=\left(\begin{array}{cc}
\zeta_{p}^{t+1} & 0 \\
0 & \zeta_{p}^{p-t}
\end{array}\right), t=0, \ldots, p-2 .
$$

If $t=0$ then $x$ belongs to a smooth fixed curve for $\sigma$, otherwise $x$ is an isolated fixed point. We will say that an isolated point $x \in X^{\sigma}$ is of type $t(t>0)$ if the local action at $x$ is given by $A_{p, t}$ and we will denote by $n_{t}$ the number of isolated points of $\sigma$ of type $t$.

Lemma 2.2. The fixed locus of $\sigma$ is either empty or the disjoint union of isolated points and smooth curves. Moreover, in the second case, $X^{\sigma}$ is either the union of two disjoint elliptic curves or of the form

$$
X^{\sigma}=C \cup R_{1} \cup \cdots \cup R_{k} \cup\left\{p_{1}, \ldots, p_{n}\right\},
$$


where $C$ is a smooth curve of genus $g \geq 0, R_{i}$ is a smooth rational curve and $p_{i}$ is an isolated point.

Proof. The first statement follows from the previous discussion about the local action of $\sigma$. By Hodge index theorem the Picard lattice of $X$ is hyperbolic. Thus, if $X^{\sigma}$ contains a smooth curve $C$ of genus $g>1$, then the other curves in the fixed locus are rational (by adjunction formula, since their class have negative selfintersection).

If $X^{\sigma}$ contains an elliptic curve, then the other fixed curves can be either rational or elliptic. Assume that there are two fixed elliptic curves $E_{1}, E_{2}$. Since $E_{1}, E_{2}$ are disjoint, then their classes are linearly equivalent and define a $\sigma$-invariant elliptic fibration $\varphi: X \rightarrow \mathbb{P}^{1}$. Since the local action of $\sigma$ at $p \in E_{1}$ is of type $(x, y) \mapsto\left(x, \zeta_{p} y\right)$, then $\sigma$ induces a non-trivial action on $\mathbb{P}^{1}$. Thus $\sigma$ has exactly two fixed points in $\mathbb{P}^{1}$ and its fixed locus is equal to $E_{1} \cup E_{2}$. This concludes the proof.

The aim of the rest of this section is to relate the topological invariants $g, k, n$ of the fixed locus $X^{\sigma}$ to the lattice invariants $m, a$ (and so $r, a$ ) of $T(\sigma)$. The methods we will apply generalize techniques in [10] and [20].

Lemma 2.3. The Euler characteristic of $X^{\sigma}$ is $24-m p$.

Proof. By the topological Lefschetz formula we have:

$$
\chi\left(X^{\sigma}\right)=\sum_{i=0}^{4}(-1)^{i} \operatorname{tr}\left(\sigma^{*} \mid H^{i}(X, \mathbb{R})\right)=2+\operatorname{tr}\left(\sigma^{*} \mid S(\sigma)\right)+\operatorname{tr}\left(\sigma^{*} \mid T(\sigma)\right)=2+r-m,
$$

where $r$ is the rank of $S(\sigma)$. This gives the statement since $m(p-1)=22-r$.

Observe that, by Lemma 2.2, the Euler characteristic of $X^{\sigma}$ is either zero or $\mathcal{X}\left(X^{\sigma}\right)=(2-2 g)+2 k+n$. In the second case, let $\alpha=1-g+k$.

Theorem 2.4. Let $\sigma$ be a non-symplectic automorphism of prime order $p$ of a K3 surface and let $r$ be the rank of its invariant lattice $S(\sigma)$. Then the types $n_{i}$ of the isolated fixed points of $\sigma$ and the integer $\alpha$ can be expressed in function of $r$ as in Table 1.

Proof. The holomorphic Lefschetz formula [3, Theorem 4.6] allows to compute the holomorphic Lefschetz number $L(\sigma)$ of $\sigma$ in two ways. First we have that

$$
L(\sigma)=\sum_{i=0}^{2}(-1)^{i} \operatorname{tr}\left(\sigma^{*} \mid H^{i}\left(X, \mathcal{O}_{X}\right)\right) .
$$

By Serre duality $H^{2}\left(X, \mathcal{O}_{X}\right) \simeq H^{0}\left(X, \mathcal{O}_{X}\left(K_{X}\right)\right)^{\vee}$, so that

$$
L(\sigma)=1+\zeta_{p}^{p-1} .
$$

On the other hand, if the fixed locus is as in (11), we also have that

$$
L(\sigma)=\sum_{t=1}^{p-2} n_{t} a(t)+b(g)+k b(0)
$$

where

$$
a(t)=\frac{1}{\operatorname{det}\left(I-\sigma^{*} \mid T_{t}\right)}=\frac{1}{\operatorname{det}\left(I-A_{p, t}\right)}=\frac{1}{\left(1-\zeta^{t}\right)\left(1-\zeta^{p-t+1}\right)},
$$




\begin{tabular}{|c||c||c|c|c|c|c|c|c|c|c|c|}
\hline$p$ & $\alpha$ & $n_{1}$ & $n_{2}$ & $n_{3}$ & $n_{4}$ & $n_{5}$ & $n_{6}$ & $n_{7}$ & $n_{8}$ & $n_{9}$ & $n$ \\
\hline 2 & $r-10$ & & & & & & & & & & 0 \\
3 & $\frac{r-8}{2}$ & $\alpha+3$ & & & & & & & & & $\alpha+3$ \\
5 & $\frac{r-6}{4}$ & $2 \alpha+3$ & $1+\alpha$ & & & & & & & & $3 \alpha+4$ \\
7 & $\frac{r-4}{6}$ & $2 \alpha+2$ & $1+2 \alpha$ & $\alpha$ & & & & & & & $5 \alpha+3$ \\
11 & $\frac{r-2}{10}$ & $1+2 \alpha$ & $2 \alpha$ & $2 \alpha$ & $1+2 \alpha$ & $\alpha$ & & & & & $9 \alpha+2$ \\
13 & $\frac{r+2}{12}$ & $1+2 \alpha$ & $1+2 \alpha$ & $2 \alpha$ & $2 \alpha-1$ & $2 \alpha-2$ & $\alpha-1$ & & & & $11 \alpha-2$ \\
17 & $\frac{r-6}{16}$ & $2 \alpha$ & $2 \alpha$ & $2 \alpha$ & $2 \alpha$ & $2 \alpha+1$ & $2 \alpha+2$ & $2 \alpha+3$ & $\alpha+1$ & & $15 \alpha+7$ \\
19 & $\frac{r-4}{18}$ & $2 \alpha$ & $2 \alpha$ & $2 \alpha$ & $2 \alpha+1$ & $2 \alpha+2$ & $2 \alpha+1$ & $2 \alpha+1$ & $2 \alpha$ & $\alpha$ & $17 \alpha+5$ \\
\hline
\end{tabular}

TABLE 1. Isolated fixed points

with $T_{t}$ the tangent space of $X$ at a point of type $t$, and

$$
b(g)=\frac{1-g}{1-\zeta_{p}}-\frac{\zeta_{p}(2 g-2)}{\left(1-\zeta_{p}\right)^{2}}=\frac{\left(1+\zeta_{p}\right)(1-g)}{\left(1-\zeta_{p}\right)^{2}}, \quad b(0)=\frac{1+\zeta_{p}}{\left(1-\zeta_{p}\right)^{2}} .
$$

If $X^{\sigma}$ is either empty or the union of two elliptic curves, then $L(\sigma)=0$.

Combining (2), (3) and (4) we get the types $n_{i}$ appearing in Table 1 Moreover, since $\chi\left(X^{\sigma}\right)=2 \alpha+n$, we get the values of $\alpha$ in Table 1 by applying Lemma 2.3 ,

We now assume that the fixed locus is as in (1) and we will determine the genus $g$ as a function of the invariants $m, a$ of $T(\sigma)$ by means of Smith exact sequences. We will consider the following isometries of $H^{2}(X, \mathbb{Z})$ :

$$
\mathfrak{g}=1+\sigma^{*}+\left(\sigma^{*}\right)^{2}+\cdots+\left(\sigma^{*}\right)^{p-1}, \quad \mathfrak{h}=1-\sigma^{*} .
$$

Observe that $\operatorname{ker} \mathfrak{h}=S(\sigma)$, ker $\mathfrak{g}=T(\sigma)$ and $\left|H^{2}(X, \mathbb{Z}) / S(\sigma) \oplus T(\sigma)\right|=p^{a}$. We now consider the coefficient homomorphism

$$
c: H^{2}(X, \mathbb{Z}) \longrightarrow H^{2}\left(X, \mathbb{Z}_{p}\right) .
$$

Observe that $c(S(\sigma) \oplus T(\sigma))$ coincides with $E=\operatorname{ker} \mathfrak{g} \subset H^{2}\left(X, \mathbb{Z}_{p}\right)$. This implies that $a=\operatorname{dim} H^{2}\left(X, \mathbb{Z}_{p}\right)-\operatorname{dim} E$.

Let $C(X)$ be the chain complex of $X$ with coefficients in $\mathbb{Z}_{p}$. The automorphism $\sigma$ acts on $C(X)$ and gives rise to chain subcomplexes $\mathfrak{g} C(X)$ and $\mathfrak{h} C(X)$. We denote by $H_{i}^{\mathfrak{g}}(X), H_{i}^{\mathfrak{h}}(X)$ the associated Smith special homology groups with coefficients in $\mathbb{Z}_{p}$ as in [6. Definition 3.2, Ch. III] and by $\chi^{\mathfrak{g}}(X), \chi^{\mathfrak{h}}(X)$ the corresponding Euler characteristics. By [6, (3.4), Ch. III] there is an isomorphism

$$
H_{i}^{\mathfrak{g}}(X) \simeq H_{i}\left(X /\langle\sigma\rangle, X^{\sigma}\right),
$$

where $X^{\sigma}$ is identified with its image in the quotient surface $X /\langle\sigma\rangle$.

In what follows, the coefficients are intended to be in $\mathbb{Z}_{p}$. Observe that $\mathfrak{g}=\mathfrak{h}^{p-1}$ over $\mathbb{Z}_{p}$. Let $\rho=\mathfrak{h}^{i}$ and $\bar{\rho}=\mathfrak{h}^{p-i}$, then for any $i, j=1, \ldots, p-1$ we have the exact 
triangles ([6, Theorem 3.3 and (3.8), Ch. III])
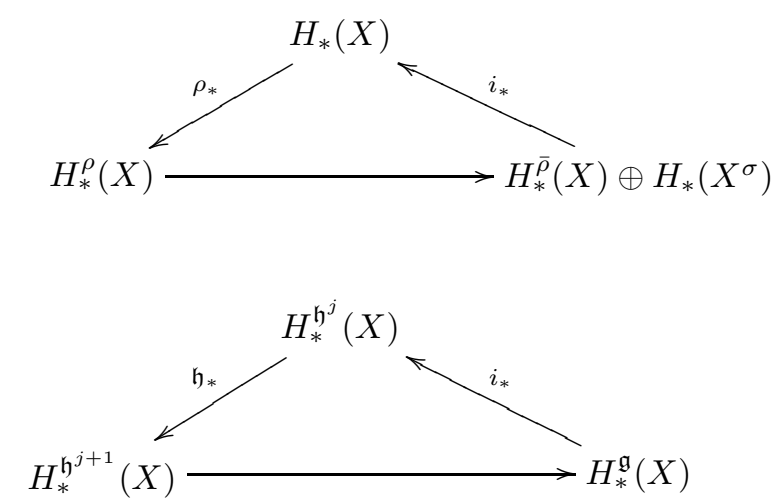

where $\mathfrak{h}_{*}, i_{*}$ and $\rho_{*}$ have degree 0 and the horizontal arrows have degree -1 . The triangle (T1) induces two long homology sequences for $\rho=\mathfrak{g}$ and $\mathfrak{h}$. In particular, since $H_{1}(X)=H_{3}(X)=0$, then (T1) induces the sequences:

$$
\begin{aligned}
0 & \rightarrow H_{3}^{\mathfrak{g}}(X) \stackrel{\gamma_{3}}{\rightarrow} H_{2}^{\mathfrak{h}}(X) \oplus H_{2}\left(X^{\sigma}\right) \stackrel{\alpha_{2}}{\rightarrow} H_{2}(X) \stackrel{\beta_{2}}{\rightarrow} H_{2}^{\mathfrak{g}}(X) \stackrel{\gamma_{2}}{\rightarrow} H_{1}^{\mathfrak{h}}(X) \oplus H_{1}\left(X^{\sigma}\right) \rightarrow 0, \\
0 & \rightarrow H_{3}^{\mathfrak{h}}(X) \stackrel{\gamma_{3}^{\prime}}{\rightarrow} H_{2}^{\mathfrak{g}}(X) \oplus H_{2}\left(X^{\sigma}\right) \stackrel{\alpha_{2}^{\prime}}{\rightarrow} H_{2}(X) \stackrel{\beta_{2}^{\prime}}{\rightarrow} H_{2}^{\mathfrak{h}}(X) \stackrel{\gamma_{2}^{\prime}}{\rightarrow} H_{1}^{\mathfrak{g}}(X) \oplus H_{1}\left(X^{\sigma}\right) \rightarrow 0 .
\end{aligned}
$$

Lemma 2.5. $H_{0}^{\mathfrak{g}}(X)=H_{0}^{\mathfrak{h}}(X)=0, \operatorname{dim} H_{i}^{\mathfrak{h}}(X)=\operatorname{dim} H_{i}^{\mathfrak{g}}(X)=1$ for $i=1,3,4$.

Proof. Since $X /\langle\sigma\rangle$ is connected, then $H_{0}^{\mathfrak{g}}(X) \simeq H_{0}\left(X /\langle\sigma\rangle, X^{\sigma}\right)=0$ (see [9, Prop. 13.10]). This implies that $\operatorname{dim} H_{0}^{\mathfrak{h}}(X)=0$ by repeated use of (T2). By (T1), this gives $\operatorname{dim} H_{1}^{\mathfrak{h}}(X)=\operatorname{dim} H_{1}^{\mathfrak{g}}(X)$.

Moroever, by (T1) we get $\operatorname{dim} H_{3}^{\mathfrak{h}}(X)=\operatorname{dim} H_{3}^{\mathfrak{g}}(X)$ and $\operatorname{dim} H_{4}^{\mathfrak{g}}(X)+\operatorname{dim} H_{4}^{\mathfrak{h}}(X)-$ $\operatorname{dim} H_{3}^{\mathfrak{g}}(X)=1$. The exact sequence of the pair $\left(X /\langle\sigma\rangle, X^{\sigma}\right)$ gives $\operatorname{dim} H_{4}^{\mathfrak{g}}(X)=$ $\operatorname{dim} H_{4}(X /\langle\sigma\rangle)=1$, which implies that $\operatorname{dim} H_{4}^{\mathfrak{h}}(X)=\operatorname{dim} H_{3}^{\mathfrak{g}}(X)=\operatorname{dim} H_{3}^{\mathfrak{h}}(X)$. Observe that $\operatorname{dim} H_{4}^{\mathfrak{h}}(X) \leq 1$ by (T1) (has an injective map to $H^{4}(X)$ ) and $\operatorname{dim} H_{4}^{\mathfrak{h}}(X) \geq 1$ by (T2) (there is an injective map from $H_{4}^{\mathfrak{g}}(X)$ to $H_{4}^{\mathfrak{h}}(X)$ ). This gives the statement.

Lemma 2.6. $i_{*}: H_{2}^{\mathfrak{g}} \rightarrow H_{2}^{\mathfrak{h}}$ is injective.

Proof. Assume that $i_{*}$ is not injective. By [6, (3.7), Ch. III] we have the following exact square:

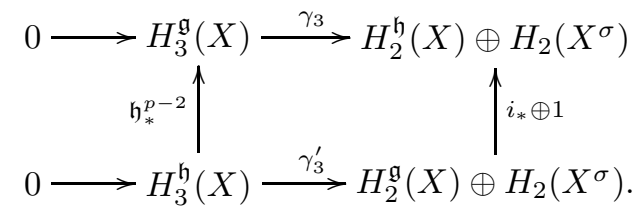

Thus $\mathfrak{h}_{*}^{p-2}$ is the zero homomorphism, since $\operatorname{dim} H_{3}^{\mathfrak{h}}(X)=\operatorname{dim} H_{3}^{\mathfrak{g}}(X)=1$ by Lemma 2.5. It can be easily seen that this leads to a contradiction looking at the first terms in the homology ladders associated to the diagrams [6, (3.6) and (3.7), Ch. III].

Lemma 2.7. $\operatorname{dimim} \alpha_{2}=\operatorname{dim} E, \operatorname{dim} i m \alpha_{2}-\operatorname{dimim} \alpha_{2}^{\prime}=\operatorname{dim} H_{2}^{\mathfrak{h}}(X)-\operatorname{dim} H_{2}^{\mathfrak{g}}(X)$. 
Proof. We will denote by $E^{\prime}=\operatorname{ker} \mathfrak{g}_{*} \subset H_{2}(X)$. Observe that $E^{\prime}$ is the dual of $E$, so that $\operatorname{dim} E=\operatorname{dim} E^{\prime}$.

We will use the following properties of Smith exact sequences: $\operatorname{im} \alpha_{2} \subset E^{\prime}$ and the projection of $\gamma_{3}$ on the second factor is the boundary homomorphism in the sequence of the relative homology $H_{i}\left(X /\langle\sigma\rangle, X^{\sigma}\right)$. The latter property implies that such projection is injective, since $H_{3}(X /\langle\sigma\rangle)=0$

If $x \in E^{\prime}$ then $\alpha_{2}^{\prime}\left(\beta_{2}(x) \oplus 0\right)=\mathfrak{g}_{*}(x)=0$. Hence $\beta_{2}(x) \oplus 0 \in \operatorname{ker} \alpha_{2}^{\prime}=\operatorname{im} \gamma_{3}^{\prime}$. By the square (5), we get that $i_{*}\left(\beta_{2}(x)\right) \oplus 0=\gamma_{3}(y) \in \operatorname{im}\left(\gamma_{3}\right)$. Since the projection of $\gamma_{3}$ on the second factor is injective, then $\gamma_{3}(y)=i_{*}\left(\beta_{2}(x)\right)=0$. By Lemma 2.6. this implies that $x \in \operatorname{ker}\left(\beta_{2}\right)=\operatorname{im}\left(\alpha_{2}\right)$, proving that $E^{\prime} \subset \operatorname{im} \alpha_{2}$.

The two exact sequences induced by (T1) and Lemma 2.5 give the second statement.

Proposition 2.8. $\sum_{i} \operatorname{dim} H_{i}\left(X^{\sigma}, \mathbb{Z}_{p}\right)=24-2 a-m(p-2)$.

Proof. By (T2) we have $\chi^{\mathfrak{h}^{j}}(X)=\chi^{\mathfrak{g}}(X)+\chi^{\mathfrak{h}^{j+1}}(X)$ for any $j=1, \ldots, p-1$. Since $\mathfrak{h}^{p-1}=\mathfrak{g}$, then $\chi^{\mathfrak{h}}(X)=(p-1) \chi^{\mathfrak{g}}(X)$. Moreover, by (T1) we get:

$$
\chi(X)-\chi\left(X^{\sigma}\right)=\chi^{\mathfrak{g}}(X)+\chi^{\mathfrak{h}}(X)=p \chi^{\mathfrak{g}}(X) .
$$

Thus by Lemma 2.3 we have

$$
\chi^{\mathfrak{g}}(X)=m \text {. }
$$

On the other hand $\chi^{\mathfrak{g}}(X)-\chi^{\mathfrak{h}}(X)=\operatorname{dim} H_{2}^{\mathfrak{g}}(X)-\operatorname{dim} H_{2}^{\mathfrak{h}}(X)$ by Lemma 2.5 Hence, by Lemma 2.7, we get

$$
\operatorname{dimim} \alpha_{2}-\operatorname{dimim} \alpha_{2}^{\prime}=\chi^{\mathfrak{h}}(X)-\chi^{\mathfrak{g}}(X)=\chi^{\mathfrak{g}}(X)(p-2)=m(p-2) .
$$

Then from the two exact sequences, Lemma 2.5 and 2.7 we have

$$
\begin{aligned}
& \sum_{i} \operatorname{dim} H_{i}(X)-\sum_{i} \operatorname{dim} H_{i}\left(X^{\sigma}\right)=\chi^{\mathfrak{g}}(X)+\chi^{\mathfrak{h}}(X)-2 \operatorname{dim} H_{1}\left(X^{\sigma}\right) \\
& =\operatorname{dim} \operatorname{im} \beta_{2}+\operatorname{dim} \operatorname{im} \beta_{2}^{\prime}=2\left(\operatorname{dim} H_{2}(X)-\operatorname{dim} \operatorname{im} \alpha_{2}\right)+\operatorname{dim} \operatorname{im} \alpha_{2}-\operatorname{dim} \operatorname{im} \alpha_{2}^{\prime} \\
& =2 a+m(p-2) .
\end{aligned}
$$

This concludes the proof since $\sum_{i} \operatorname{dim} H_{i}(X)=24$.

Corollary 2.9. If the fixed locus of $\sigma$ is as in (1), then $2 g=m-a$. Otherwise, if it is either empty or the union of two elliptic curves, then $m=a$ and $m-a=4$ respectively.

Proof. It follows from Lemma 2.3 and Proposition 2.8, since

$$
\sum_{i} \operatorname{dim} H_{i}\left(X^{\sigma}, \mathbb{Z}_{p}\right)-\chi\left(X^{\sigma}, \mathbb{Z}_{p}\right)=2 \operatorname{dim} H_{1}\left(X^{\sigma}, \mathbb{Z}_{p}\right)=4 g
$$

in case the fixed locus is as in (10) (and equals 0 and 8 if it is empty or the union of two elliptic curves respectively).

Remark 2.10. By Corollary 2.9 the integer $m-a$ is positive and even, this is equivalent to the condition $(*)$ in Theorem 0.1 .

Moreover, by Lemma 2.3. Theorem 2.4 and Corollary 2.9 it follows that the invariants $g, k, n$ of the fixed locus of $\sigma$ uniquely determine the invariants $r, a$ (or $m, a)$ of the invariant lattice and viceversa. Thus, by Theorem 1.1, it is equivalent to give the topology of the fixed locus of $\sigma$ or its invariant lattice in $H^{2}(X, \mathbb{Z})$ if $p \geq 3$. An easy computation gives the formulas for $n, k$ as functions of $r, a$ given in Theorem 0.1 . 


\section{ORDER 2}

We briefly recall the classification theorem for non-symplectic involutions on $K 3$ surfaces given by Nikulin in [20, §4] and [21, §4]. The local action of a nonsymplectic involution $\sigma$ at a fixed point is of type

$$
A_{2,0}=\left(\begin{array}{cc}
1 & 0 \\
0 & -1
\end{array}\right)
$$

so that $X^{\sigma}$ is the disjoint union of smooth curves and there are no isolated fixed points. The lattice $S(\sigma)$ is 2-elementary thus, according to Theorem 1.1, its isometry class is determined by the invariants $r, a$ and $\delta$.

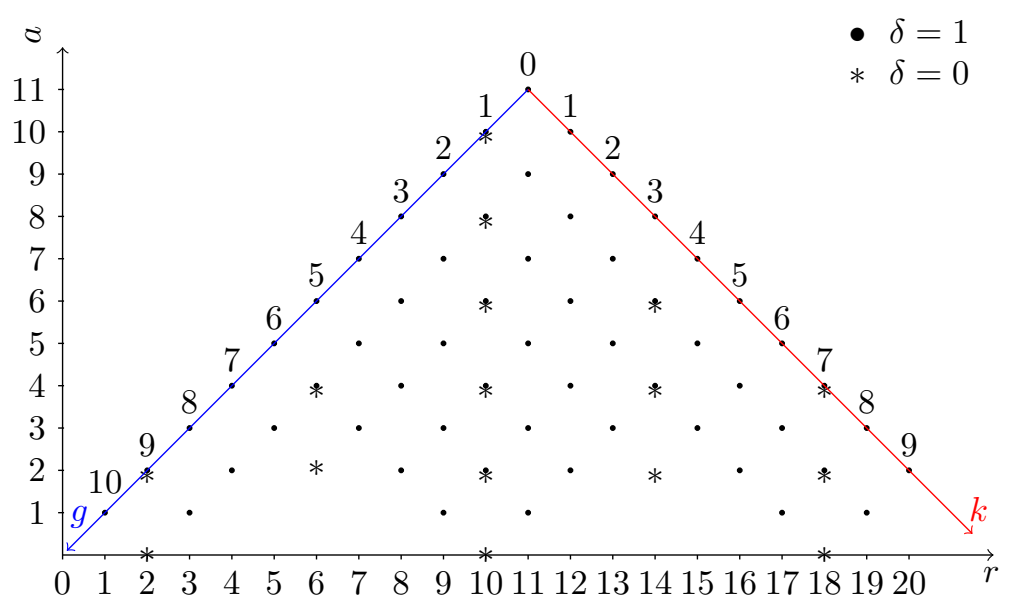

Figure 1. Order 2

Theorem 3.1 (Theorem 4.2.2, 20]). The fixed locus of a non-symplectic involution on a $K 3$ surface is

- empty if $r=10, a=10$ and $\delta=0$,

- the disjoint union of two elliptic curves if $r=10, a=8$ and $\delta=0$,

- the disjoint union of a curve of genus $g$ and $k$ rational curves otherwise, where

$$
g=(22-r-a) / 2, \quad k=(r-a) / 2 .
$$

Figure 1 shows all the values of the triple $(r, a, \delta)$ which are realized and the corresponding invariants $(g, k)$ of the fixed locus.

The surfaces which arise as quotients of $K 3$ surfaces by non-symplectic involutions have been classified in [21, 1, 33]. These are Enriques surfaces if $X^{\sigma}=\emptyset$ and smooth rational surfaces otherwise.

\section{ORDER 3}

Non-symplectic automorphisms of order 3 on K3 surfaces have been recently classified in [2] and [31]. In this case the local action at a fixed point is of one of the following

$$
A_{3,1}=\left(\begin{array}{cc}
\zeta_{3}^{2} & 0 \\
0 & \zeta_{3}^{2}
\end{array}\right), \quad A_{3,0}=\left(\begin{array}{cc}
\zeta_{3} & 0 \\
0 & 1
\end{array}\right)
$$


so that the fixed locus contains both smooth curves and isolated points. Let $(r, a)$ be the invariants of $S(\sigma)$, and let $\operatorname{rank} T(\sigma)=2 m$ as in section $\S 3$.

Theorem 4.1 (Table 1, [2] and Theorem 1.2, 31]). The fixed locus of a nonsymplectic automorphism of order 3 on a $K 3$ surface is not empty and it is either:

- the union of three isolated points if $m=a=7$, or

- the disjoint union of $n$ points, a smooth curve of genus $g$ and $k$ smooth rational curves, where

$$
n=10-m, \quad g=(m-a) / 2, \quad k=6-(m+a) / 2 .
$$

All values of the pair $(m, a)$ and the corresponding invariants $(g, k, n)$ of the fixed locus are represented in Figure 2 .

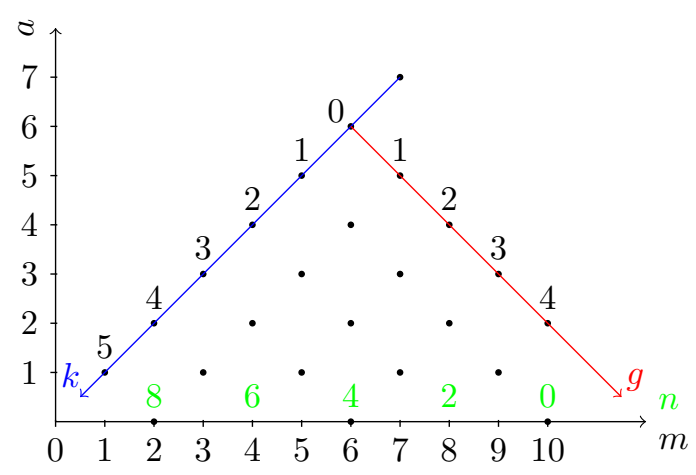

FiguRE 2. Order 3

Remark 4.2. We give here a construction relating $K 3$ surfaces with $n=3, k=0$, $g=1$ to those with $n=3$ and no fixed curves (cf. [2, Proposition 4.7]). Consider the elliptic $K 3$ surface $X_{a, b}$ defined by the Weierstrass equation

$$
y^{2}=x^{3}+\left(t^{6}+a_{1} t^{3}+a_{2}\right) x+\left(t^{12}+b_{1} t^{9}+b_{2} t^{6}+b_{3} t^{3}+b_{4}\right),
$$

where $a \in \mathbb{C}^{2}, b \in \mathbb{C}^{4}$ are generic. The fibration has 24 fibers of Kodaira type $I_{1}$ over the zeros of its discriminant polynomial. The automorphism of order 3

$$
\sigma(x, y, t)=\left(x, y, \zeta_{3} t\right)
$$

acts non trivially on the basis of the fibration and preserves the smooth elliptic curves over $t=0$ and $t=\infty$. Moreover, the fiber over $t=0$ is pointwise fixed. This implies that $\sigma$ is non-symplectic and by Theorem 4.1 we have $n=3, k=0$, $g=1$. The minimal resolution of the quotient surface $X_{a, b} /\langle\sigma\rangle$ is a rational elliptic surface $\pi_{a, b}: Y_{a, b} \rightarrow \mathbb{P}^{1}$ with Weierstrass equation

$$
y^{2}=x^{3}+\left(t^{2}+a_{1} t+a_{2}\right) x+\left(t^{4}+b_{1} t^{3}+b_{2} t^{2}+b_{3} t+b_{4}\right) .
$$

This fibration has one singular fiber of Kodaira type $I V$ over $t=\infty$ and 8 fibers of type $I_{1}$. Let $p: P \rightarrow \mathbb{P}^{1}$ be a non-trivial principal homogeneous space of $\pi_{a, b}$ given by an order 3 element in the fiber $\pi_{a, b}^{-1}(0)$ (see [7, Ch.V, $\left.\S 4\right]$ ). Then surface $P$ is a rational elliptic surface with a multiple smooth elliptic fiber of multiplicity 3 over 0 . Let $Z$ be the surface obtained by blowing up the intersection point of the three rational curves of the fiber $p^{-1}(\infty)$ and then blowing down the strict transforms of 
the three rational curves. Then $Z$ is a $\log$ Enriques surface of index 3 with three singular points. Let $X$ be the canonical cover of $Z$ and $\bar{\sigma}$ be a generator of the covering transformation group of the cover. By [35, Theorem 5.1], since $Z$ has three singular points of type $(3,1)$ (these are Hirzebruch-Jung singularities, cf. 4, Section 5]) then $X$ is a $K 3$ surface and $\bar{\sigma}$ is an order 3 non-symplectic automorphism of $X$ with only three fixed points.

A similar construction relates Examples [5.6, 6.1, 7.1 to Examples 5.8, 6.2, 7.2 respectively.

\section{ORDER 5}

An order five non-symplectic automorphism of a $K 3$ surface has three possible local actions at a fixed point

$$
A_{5,0}=\left(\begin{array}{cc}
\zeta_{5} & 0 \\
0 & 1
\end{array}\right), \quad A_{5,2}=\left(\begin{array}{cc}
\zeta_{5}^{3} & 0 \\
0 & \zeta_{5}^{3}
\end{array}\right), \quad A_{5,1}=\left(\begin{array}{cc}
\zeta_{5}^{2} & 0 \\
0 & \zeta_{5}^{4}
\end{array}\right) .
$$

Thus the fixed locus can contain both fixed curves and isolated points of two different types. We start providing two families of examples.

Example 5.1. Let $\mathcal{A}$ be the family of plane sextic curves defined by

$$
x_{0}\left(x_{0}-x_{1}\right) \prod_{i=1}^{4}\left(x_{0}-\lambda_{i} x_{1}\right)+x_{2}^{5} x_{1}=0,
$$

where $\lambda_{i} \in \mathbb{C}$. Observe that the projective transformation

$$
\bar{\sigma}\left(x_{0}, x_{1}, x_{2}\right)=\left(x_{0}, x_{1}, \zeta_{5} x_{2}\right)
$$

preserves any curve in $\mathcal{A}$. If $C \in \mathcal{A}$ is smooth, then the double cover $X$ of $\mathbb{P}^{2}$ branched along $C$ is a $K 3$ surface and $\bar{\sigma}$ induces an automorphism $\sigma$ of order 5 on $X$. Since $X^{\bar{\sigma}}=\{(0,0,1)\} \cup\left\{x_{2}=0\right\}$, then $X^{\sigma}$ is the union of an isolated fixed point and a smooth curve of genus two. This implies that $\sigma$ is non-symplectic (since symplectic automorphisms only have isolated fixed points)

If the complex numbers $\lambda_{i}$ 's are distinct, then the corresponding sextic $C \in \mathcal{A}$ is smooth and has six fixed points on the line $x_{2}=0$. Otherwise, if two or three of the $\lambda_{i}$ 's coincide, then $C$ has a singular point of type $A_{4}$ or $E_{8}$ respectively.

More in detail, we have the following cases:

\section{Equation of $C$ :}

a) $\quad x_{0}^{2}\left(x_{0}-x_{1}\right) \prod_{i=2}^{4}\left(x_{0}-\lambda_{i} x_{1}\right)+x_{2}^{5} x_{1}=0$

b) $x_{0}^{3}\left(x_{0}-x_{1}\right)\left(x_{0}-\lambda_{3} x_{1}\right)\left(x_{0}-\lambda_{4} x_{1}\right)+x_{2}^{5} x_{1}=0$

c) $x_{0}^{2}\left(x_{0}-x_{1}\right)^{2}\left(x_{0}-\lambda_{3} x_{1}\right)\left(x_{0}-\lambda_{4} x_{1}\right)+x_{2}^{5} x_{1}=0$

d) $\quad x_{0}^{3}\left(x_{0}-x_{1}\right)^{2}\left(x_{0}-\lambda_{4} x_{1}\right)+x_{2}^{5} x_{1}=0$

e)

$$
x_{0}^{3}\left(x_{0}-x_{1}\right)^{3}+x_{2}^{5} x_{1}=0
$$

Types of singular points:

Example 5.2. Let $\mathcal{B}$ be the family of plane sextic curves defined by

$$
a_{1} x_{0}^{6}+a_{2} x_{0}^{3} x_{1} x_{2}^{2}+a_{3} x_{0}^{2} x_{1}^{3} x_{2}+x_{0}\left(a_{4} x_{1}^{5}+a_{5} x_{2}^{5}\right)+a_{6} x_{1}^{2} x_{2}^{4}=0,
$$

where $a \in \mathbb{C}^{6}$. The projective transformation

$$
\bar{\sigma}\left(x_{0}, x_{1}, x_{2}\right)=\left(x_{0}, \zeta_{5} x_{1}, \zeta_{5}^{2} x_{2}\right)
$$




\begin{tabular}{c|c|c|c|c|c}
$n_{1}$ & $n_{2}$ & $g$ & $k$ & $T(\sigma)$ & $S(\sigma)$ \\
\hline 1 & 0 & 2 & 0 & $H_{5} \oplus U \oplus E_{8} \oplus E_{8}$ & $H_{5}$ \\
\hline 3 & 1 & 1 & 0 & $H_{5} \oplus U \oplus E_{8} \oplus A_{4}$ & $H_{5} \oplus A_{4}$ \\
3 & 1 & - & - & $H_{5} \oplus U(5) \oplus E_{8} \oplus A_{4}$ & $H_{5} \oplus A_{4}^{*}(5)$ \\
\hline 5 & 2 & 1 & 1 & $U \oplus H_{5} \oplus E_{8}$ & $H_{5} \oplus E_{8}$ \\
5 & 2 & 0 & 0 & $U \oplus H_{5} \oplus A_{4}^{2}$ & $H_{5} \oplus A_{4}^{2}$ \\
\hline 7 & 3 & 0 & 1 & $U \oplus H_{5} \oplus A_{4}$ & $H_{5} \oplus A_{4} \oplus E_{8}$ \\
\hline 9 & 4 & 0 & 2 & $U \oplus H_{5}$ & $H_{5} \oplus E_{8} \oplus E_{8}$
\end{tabular}

TABLE 2. Order 5

preserves any curve in $\mathcal{B}$. If $C \in \mathcal{B}$ is smooth, then the double cover $X$ of $\mathbb{P}^{2}$ branched along $C$ is a $K 3$ surface and $\bar{\sigma}$ induces a non-symplectic automorphism $\sigma$ of order 5 on $X$. In fact $\sigma$ does not leave invariant the holomorphic 2-form (written in local coordinates):

$$
\frac{d x \wedge d y}{\sqrt{f}}
$$

where $f$ denotes the equation of a curve in $\mathcal{B}$ in local coordinates $x, y$. Since $X^{\bar{\sigma}}=\{(1,0,0)\} \cup\{(0,1,0)\} \cup\{(0,0,1)\}$ and all but one of these points belong to $C$, then $X^{\sigma}$ is the union of 4 isolated points.

Theorem 5.3. The fixed locus of a non-symplectic automorphism $\sigma$ of order 5 on a K3 surface is either

- the union of three isolated points of type 1 and one point of type 2 if $S(\sigma)$ is isomorphic to $H_{5} \oplus A_{4}^{*}(5)$, or

- the disjoint union of $n_{i}$ isolated points of type $i$, a smooth curve of genus $g$ and $k$ smooth rational curves where $\left(g, k, n_{i}\right)$ appears in a row of Table Q.

The same table gives the corresponding invariant lattice $S(\sigma)$ and its orthogonal complement $T(\sigma)$.

Proof. The list of lattices in Table 2 is obtained by using Theorem 1.1 and Corollary 2.9 (which implies that $m-a$ is a positive even integer). For any such lattice $S(\sigma)$ the invariants $g, k, n_{i}$ of $X^{\sigma}$ can be computed by means of Lemma 2.3. Theorem 2.4 and Corollary 2.9.

We will now show that a $K 3$ surface $X$ with a non-symplectic automorphism $\sigma$ of order 5 such that $S_{X}=S(\sigma)$ belongs to one of the two families in Examples 5.1. 5.2. This will also show that all cases in Table 2 do appear.

By Theorem 5.3 the invariant lattice of $\sigma$ is one of the following types.

$S(\sigma) \cong H_{5}$ Let $h$ be the generator of $H_{5}$ with $h^{2}=2$. By [14, Lemma 3.5] we can assume $h$ to be ample and base point free. Thus the morphism associated to $|h|$ is a double cover of $\mathbb{P}^{2}$ branched along a smooth plane sextic $B$. Moreover, since $h \in S(\sigma)$, then the action of $\sigma$ on $X$ induces a projectivity $\bar{\sigma}$ of $\mathbb{P}^{2}$. By Theorem 5.3 the fixed locus of $\sigma$ contains a curve of genus $g=2$. This implies that $\bar{\sigma}$ has a curve in the fixed locus, hence for a suitable choice of coordinates it is of the form 
(6). Thus $(X, \sigma)$ belongs to the family in Example 5.1 .

$S(\sigma) \cong H_{5} \oplus A_{4}$ In this case we can assume $h$ to be nef and base point free, by a reasoning similar to the one in the proof of 14, Lemma 3.5]. The associated morphism is a double cover of $\mathbb{P}^{2}$ branched along a plane sextic $B$ with a singular point $p$ of type $A_{4}$. By Theorem 5.3 the fixed locus of $\sigma$ contains a fixed curve. As before, this implies that $\bar{\sigma}$ is of type (6). This implies that $X$ belongs to the family in Example 5.1, a).

The cases $H_{5} \oplus E_{8}, H_{5} \oplus A_{4}^{2}, H_{5} \oplus A_{4} \oplus E_{8}$ and $H_{5} \oplus E_{8}^{2}$ can be discussed in a similar way and correspond to Examples $5.1 c$ ),$d$ ) and $e$ ) respectively.

$S(\sigma) \cong H_{5} \oplus A_{4}^{*}(5)$ Since $A_{4}^{*}(5)$ does not contain vectors with self-intersection -2 (see the description of $A_{4}^{*}$ in [30]), then we can assume $h$ to be ample as before and the associated morphism is a double cover branched along a smooth plane sextic $B$. By Theorem 5.3, the automorphism $\sigma$ has at most isolated fixed points. Thus the same is true for $\bar{\sigma}$ so that, for a suitable choice of coordinates, it is of the form (77). Hence $(X, \sigma)$ is as in Example 5.2.

Remark 5.4. In 24] K. Oguiso and D-Q. Zhang showed the uniqueness of a $K 3$ surface with a non-symplectic automorphism of order five and fixed locus containing no curves of genus $\geq 2$ and at least 3 rational curves. In their approach they used $\log$ Enriques surfaces.

Remark 5.5. In [12, $§ 3.1,3.2]$ and in [13, Remark 6] S. Kondō considers the minimal resolution $X$ of the double cover of $\mathbb{P}^{2}$ branched along the union of the line $x_{2}=0$ and the plane quintic curve

$$
x_{2}^{5}=\prod_{i=1}^{5}\left(x_{0}-\lambda_{i} x_{1}\right),
$$

where the $\lambda_{i}$ 's are distinct complex numbers. Then $X$ is a $K 3$ surface with an automorphism $\sigma$ induced from $\bar{\sigma}$ as in (6) with $n=7, g=0, k=0$ and $T(\sigma) \cong$ $U \oplus H_{5} \oplus A_{4}^{2}$ as computed in [8, Section 12 , p. 53]. Since this family has dimension 2 , this gives a different model for the family of $K 3$ surfaces in Example 5.1 c).

In [11, $\S 7,(7.6)]$ appears the following elliptic $K 3$ surface with order 5 automorphism:

$$
y^{2}=x^{3}+t^{3} x+t^{7}, \quad \sigma(x, y, t)=\left(\zeta_{5}^{3} x, \zeta_{5}^{2} y, \zeta_{5}^{2} t\right) .
$$

Here the fixed locus has the invariants $n=13, g=0$ and $k=2$, hence this gives a different model for Example 5.1, e).

Example 5.6. Let $X_{\alpha, \beta, \gamma}$ be an elliptic $K 3$ surface with Weierstrass equation

$$
y^{2}=x^{3}+\left(t^{5}+\alpha\right) x+\left(\beta t^{10}+t^{5}+\gamma\right), \quad \alpha, \beta, \gamma \in \mathbb{C} .
$$

For generic $\alpha, \beta$ and $\gamma$ the fibration has a fiber of Kodaira type $I V$ over $t=\infty$ and 20 fibers of type $I_{1}$. Observe that the fibration has an automorphism of order 5 :

$$
\sigma(x, y, t)=\left(x, y, \zeta_{5} t\right) .
$$

This automorphism acts non trivially on the basis of the fibration and preserves the fibers over $t=0$ and $t=\infty$. In fact it fixes pointwise the smooth fiber over $t=0$. This implies, by looking at the local action at a fixed point, that $\sigma$ is 
non-symplectic. Checking in Table 2 one can see that the fixed locus has $n_{1}=3$, $n_{2}=1, g=1$ and $k=0$. In fact it is easy to see that this is an equation of the generic $K 3$ surface in the family of Example 5.1, a). Moreover observe that:

1) If $\beta=0$ then the fibration has a singular fiber of Kodaira type $I I I^{*}$ over $t=\infty$ and 15 fibers of type $I_{1}$. This corresponds to the case $n_{1}=5, n_{2}=2, g=1$, $k=1$ in Table 2 ,

2) If $\alpha^{3}=-27 / 4 \gamma^{2}$ then the fibration has a singular fiber of type $I V$ over $t=\infty$ and of type $I_{5}$ over $t=0$. This corresponds to $n_{1}=5, n_{2}=2, g=k=0$.

3) If $\alpha=\gamma=0$ then there is a fiber of type $I V$ over $t=\infty$ and a fiber of type $I I^{*}$ over $t=0$. This corresponds to $n_{1}=7, n_{2}=3, g=0, k=1$.

4) If $\alpha=\beta=\gamma=0$ then there is a fiber of type $I I I^{*}$ over $t=\infty$ and a fiber of type $I I^{*}$ over $t=0$. This corresponds to $n_{1}=9, n_{2}=4, g=0, k=2$.

We recall that a log-Enriques surface is a normal algebraic surface $Y$ having at most quotient singularities such that $h^{1}\left(Y, \mathcal{O}_{Y}\right)=0$ and $m K_{Y}=\mathcal{O}_{Y}$ for some positive integer $m$. The index of $Y$ is the smallest positive $m$ with this property. The canonical covering $q: \tilde{Y} \rightarrow Y$ (i.e. that induced by the relation $m K_{Y}=\mathcal{O}_{Y}$ ) is a cyclic cover of degree $m$, étale over the smooth points of $Y$. The minimal resolution of $\tilde{Y}$ is known to be either a $K 3$ surface or an abelian surface.

Remark 5.7. The general theory of $\log$ Enriques surfaces has been developed in [34, 35, 36. Moreover, log Enriques surfaces of index 2 have been studied in [33, of index 5 in [24], of index 11 in [22], and of index 13,17,19 in [23].

Example 5.8. Let $\pi_{a, b, c}: Y_{a, b, c} \rightarrow \mathbb{P}^{1}$ be the rational jacobian elliptic surface with Weierstrass equation

$$
y^{2}=x^{3}+t x+\left(a t^{2}+b t+c\right), \quad a, b, c \in \mathbb{C} .
$$

For generic $a, b, c$ the elliptic fibration has a fiber of Kodaira type $I V^{*}$ at $t=\infty$ and 4 fibers of type $I_{1}$. Let $p: P \rightarrow \mathbb{P}^{1}$ be a non-trivial principal homogeneous space of $\pi_{a, b, c}$ given by an order 5 element in the fiber $\pi_{a, b, c}^{-1}(0)$ (see [7, Ch.V, $\left.\S 4\right]$ ). Then $P$ is a rational elliptic surface with a multiple smooth elliptic fiber of multiplicity 5 over 0 . Let $Z$ be the surface obtained by blowing up the intersection points of the component of multiplicity 3 of $p^{-1}(\infty)$ with those of multiplicity 2 and then blowing down the four connected components of the proper transform of $p^{-1}(\infty)$. Then an easy computation shows that $Z$ is a $\log$ Enriques surface of index 5 with 4 singular points (the images of the components of $p^{-1}(\infty)$ ). Let $X$ be the canonical cover of $Z$ and $\sigma$ be a generator of the covering transformation group of the cover. By [35, Theorem 5.1], since $Z$ has three singular points of type $(5,2)$ and one of type $(5,1)$, then $X$ is a $K 3$ surface. Moreover, $\sigma$ is an order 5 non-symplectic automorphism of $X$ with only isolated fixed points.

\section{ORDER 7}

The local actions of an order 7 non-symplectic automorphism on a $K 3$ surface at a fixed point are of four types

$$
A_{7,0}=\left(\begin{array}{cc}
1 & 0 \\
0 & \zeta_{7}
\end{array}\right), A_{7,1}=\left(\begin{array}{cc}
\zeta_{7}^{2} & 0 \\
0 & \zeta_{7}^{6}
\end{array}\right), A_{7,2}=\left(\begin{array}{cc}
\zeta_{7}^{3} & 0 \\
0 & \zeta_{7}^{5}
\end{array}\right), A_{7,3}=\left(\begin{array}{cc}
\zeta_{7}^{4} & 0 \\
0 & \zeta_{7}^{4}
\end{array}\right) .
$$

Example 6.1. Let $X_{a, b}$ be the elliptic $K 3$ surface with Weierstrass equation

$$
y^{2}=x^{3}+\left(a t^{7}+b\right) x+\left(t^{7}-1\right), \quad a, b \in \mathbb{C} .
$$




\begin{tabular}{c|c|c|c|c|c|c}
$n_{1}$ & $n_{2}$ & $n_{3}$ & $g$ & $k$ & $T(\sigma)$ & $S(\sigma)$ \\
\hline 2 & 1 & 0 & 1 & 0 & $U \oplus U \oplus E_{8} \oplus A_{6}$ & $U \oplus K_{7}$ \\
2 & 1 & 0 & - & - & $U(7) \oplus U \oplus E_{8} \oplus A_{6}$ & $U(7) \oplus K_{7}$ \\
\hline 4 & 3 & 1 & 1 & 1 & $U \oplus U \oplus E_{8}$ & $U \oplus E_{8}$ \\
4 & 3 & 1 & 0 & 0 & $U(7) \oplus U \oplus E_{8}$ & $U(7) \oplus E_{8}$ \\
\hline 6 & 5 & 2 & 0 & 1 & $U \oplus U \oplus K_{7}$ & $U \oplus E_{8} \oplus A_{6}$
\end{tabular}

TABLE 3. Order 7

For generic $a, b$ the fibration has one fiber of Kodaira type $I I I$ over $t=\infty$ and 21 fibers of type $I_{1}$. Observe that $X$ carries the order 7 automorphism

$$
\sigma(x, y, t)=\left(x, y, \zeta_{7} t\right) .
$$

Observe that:

1) If $b$ is generic, then $X_{0, b}$ has one singular fiber of type $I I^{*}$ over $t=\infty$ and 14 singular fibers of type $I_{1}$;

2) if $a$ is generic and $b^{3}=-27 / 4$, then $X_{a, b}$ has one singular fiber of type $I I I$ over $t=\infty$, one of type $I_{7}$ over $t=0$ and 14 fibers of type $I_{1}$;

3) if $b^{3}=-27 / 4$, then $X_{0, b}$ has one singular fiber of type $I I^{*}$ over $t=\infty$, one of type $I_{7}$ over $t=0$ and 7 of type $I_{1}$.

Example 6.2. Let $\pi_{a, b}: Y_{a, b} \longrightarrow \mathbb{P}^{1}$ be the rational jacobian elliptic surface with Weierstrass equation

$$
y^{2}=x^{3}+t x+(a t+b), \quad a, b \in \mathbb{C} .
$$

For generic $a, b$ the elliptic fibration has a fiber of Kodaira type $I I I^{*}$ at $t=\infty$ and 3 fibers of type $I_{1}$. To this rational surface we can associate a $K 3$ surface with an order seven non-symplectic automorphism having zero-dimensional fixed locus. The construction is similar to the one described in Example 5.8. Let $p: P \rightarrow \mathbb{P}^{1}$ be a non-trivial principal homogeneous space of $\pi_{a, b}$ given by an order 7 element in the fiber $\pi_{a, b}^{-1}(0)$. The surface $Z$ obtained by blowing up the intersection points of the component of multiplicity 4 of $p^{-1}(\infty)$ with those of multiplicity 3 and then blowing down the three connected components of the proper transform of $p^{-1}(\infty)$ is a $\log$ Enriques surface of index 7 with 3 singular points (the images of the components of $\left.p^{-1}(\infty)\right)$. By [35, Theorem 5.2] the canonical cover of $Z$ is a $K 3$ surface and a generator of the covering transformation group is an order 7 non-symplectic automorphism with only isolated fixed points.

Theorem 6.3. The fixed locus of a non-symplectic automorphism $\sigma$ of order 7 of a K3 surface is either

- the union of two isolated points of type 1 and one point of type 2 if $S(\sigma)$ is isomorphic to $U(7) \oplus K_{7}$, or

- the disjoint union of $n_{i}$ isolated points of type $i$, a smooth curve of genus $g$ and $k$ smooth rational curves where $\left(g, k, n_{i}\right)$ appears in a row of Table 3 .

The same table gives the corresponding invariant lattice $S(\sigma)$ and its orthogonal complement $T(\sigma)$. 
Proof. The lattices in Table 3 can be found by means of Theorem 1.1 and Theorem 2.1. For any such lattice $S(\sigma)$ the invariants $g, k, n_{i}$ of $X^{\sigma}$ can be computed by means of Lemma 2.3. Theorem 2.4 and Corollary 2.9.

We will now show that a $K 3$ surface $X$ with a non-symplectic automorphism $\sigma$ of order 7 such that $S_{X}=S(\sigma)$ belongs to one of the two families in Examples 6.1. 6.2. This will also show that all cases in Table 3 do appear.

Observe that each lattice $S(\sigma)$ in the table contains a copy of either $U$ or $U(7)$. This implies that the generic $K 3$ surface with Picard lattice isometric to $S$ has an elliptic fibration. By Theorem 6.3 the invariant lattice of $\sigma$ is of the following types.

$S(\sigma) \cong U \oplus K_{7}$ A $K 3$ surface $X$ with $S_{X}=S(\sigma)$ has a jacobian elliptic fibration with a unique reducible fiber of type $\tilde{A}_{1}$ (since $K_{7}$ contains a unique $(-2)$-curve, as can be checked directly). Observe that $\sigma$ preserves the elliptic fibration and its action on the basis of the fibration is not trivial, since otherwise a smooth fiber would have an order 7 automorphism with a fixed point in the intersection with the section. Thus $\sigma$ preserves two fibers: one of them is smooth and the other one is the reducible fiber. By Theorem 6.3 we know that $\sigma$ fixes exactly 3 isolated points and a smooth elliptic curve. This implies that the reducible fiber is of Kodaira type $I I I$ (if it were of type $I_{2}$ it should contain a fixed rational curve).

Since $X$ has a jacobian elliptic fibration with an order 7 automorphism acting non trivially on the basis, then we can write it in Weierstrass form:

$$
y^{2}=x^{3}+f(t) x+g(t),
$$

where $\sigma$ acts as $(x, y, t) \mapsto\left(x, y, \zeta_{7} t\right)$. The polynomials $f(t), g(t)$ are invariant for this action and $\operatorname{deg}(f) \leq 8, \operatorname{deg}(g) \leq 12$. Hence for a proper choice of coordinates we can assume $f(t)=\left(a t^{7}+b\right) t^{m}$ and $g(t)=\left(c t^{7}-d\right) t^{n}$ where $m \leq 1, n \leq 5$. Since the discriminant divisor is also $\sigma$-invariant and there are exactly 21 fibers of type $I_{1}$ in $\mathbb{P}^{1} \backslash\{\infty\}$, then $\Delta(t)=\delta\left(t^{7}-\alpha\right)\left(t^{7}-\beta\right)\left(t^{7}-\gamma\right)$. Looking at the coefficients of the equality $\Delta(t)=4 f(t)^{3}+27 g(t)^{2}$ we can deduce that $m=n=0$, hence the Weierstrass equation of the $K 3$ surface is of type

$$
y^{2}=x^{3}+\left(a t^{7}+b\right) x+\left(c t^{7}-d\right) .
$$

After applying a suitable change of coordinates we obtain a surface in the family of Example 6.1.

Similar remarks show that $K 3$ surfaces with $S_{X} \cong U \oplus E_{8}, U \oplus K_{7} \oplus A_{6}=$ $U(7) \oplus E_{8}$ and $U \oplus E_{8} \oplus A_{6}$ belong to the families a), b) and c) respectively in Example 6.1

$S(\sigma) \cong U(7) \oplus K_{7}$ By [26, Corollary $\left.3, \S 3\right]$ a $K 3$ surface $X$ with $S_{X}=S(\sigma)$ admits a $\sigma$-invariant elliptic fibration $\pi: X \rightarrow \mathbb{P}^{1}$.

By Theorem 6.3 we know that $\sigma$ has exactly 3 fixed points. This implies that $\pi_{1}$ has at most one reducible fiber of type $I I I$. Observe that $\sigma$ induces an order 7 automorphism on the basis of $\pi_{1}$ with two fixed points i.e. $\sigma$ preserves two fibers. We can assume them to be a smooth fiber over 0 and a fiber of type $I I I$ over $\infty$. Moreover, $\pi_{1}$ has generically 21 fibers of type $I_{1}$, divided in $3 \sigma$-orbits.

The quotient $X /\langle\sigma\rangle$ is a $\log$ Enriques surface of index 7 (see Lemma 1.7 and its proof in [25]) and $\pi_{1}$ induces and elliptic fibration $\bar{\pi}_{1}: X /\langle\sigma\rangle \rightarrow \mathbb{P}^{1}$. Let $Y$ be 


\begin{tabular}{c|c|c|c|c|c|c|}
$n_{1}=n_{4}$ & $n_{2}=n_{3}$ & $n_{5}$ & $g$ & $k$ & $T(\sigma)$ & $S(\sigma)$ \\
\hline 1 & 0 & 0 & 1 & 0 & $U \oplus U \oplus E_{8} \oplus E_{8}$ & $U$ \\
1 & 0 & 0 & - & - & $U \oplus U(11) \oplus E_{8} \oplus E_{8}$ & $U(11)$ \\
\hline 3 & 2 & 1 & 0 & 0 & $K_{11}(-1) \oplus E_{8}$ & $U \oplus A_{10}$
\end{tabular}

TABle 4. Order 11

the minimal resolution of $X /\langle\sigma\rangle$. The proper transform of $\bar{\pi}_{1}^{-1}(\infty)$ is a $(-1)$-curve. After contracting this curve we get a minimal rational elliptic surface $\tilde{\pi}_{1}: \bar{Y} \rightarrow \mathbb{P}^{1}$ with one fiber of type $7 I_{0}$, one of type $I I I^{*}$ and three of type $I_{1}$. It can be easily proved that the jacobian fibration of $\tilde{\pi}_{1}$ is as in Example 6.2.

Remark 6.4. A different model for the $K 3$ surface with $S_{X} \cong U \oplus E_{8} \oplus A_{6}$ was given by $\mathrm{S}$. Kondō in [11, $\S 7,(7.5)]$ :

$$
y^{2}=x^{3}+t^{3} x+t^{8}, \quad \sigma(x, y, t)=\left(\zeta^{3} x, \zeta y, \zeta^{2} t\right) .
$$

The singular fibers of this elliptic fibration are of type III*, of type $\mathrm{IV}^{*}$ and 7 of type $\mathrm{I}_{1}$. Moreover it follows from [29, §5] that the rank of its Mordell-Weil group is 1 .

\section{ORDER 11}

Non-symplectic automorphisms of order 11 of $K 3$ surfaces have been classified in 1999 by K. Oguiso and D-Q. Zhang [22. We provide here an alternative view of their classification.

Example 7.1. Let $X_{a}$ be the elliptic $K 3$ surface with Weierstrass equation

$$
y^{2}=x^{3}+a x+\left(t^{11}-1\right), \quad a \in \mathbb{C} .
$$

For generic $a \in \mathbb{C}$ the fibration has one fiber of Kodaira type $I I$ over $t=\infty$ and 22 fibers of type $I_{1}$. Observe that $X$ carries the order 11 automorphism

$$
\sigma(x, y, t)=\left(x, y, \zeta_{11} t\right) .
$$

If $a^{3}=-27 / 4$ then $X_{a}$ has one singular fiber of type $I I$ over $t=\infty$, of type $I_{11}$ over $t=0$ and 11 fibers of type $I_{1}$.

Example 7.2. Let $\pi_{a}: Y_{a} \longrightarrow \mathbb{P}^{1}$ be the rational jacobian elliptic surface with Weierstrass equation

$$
y^{2}=x^{3}+x+(t-a), \quad a \in \mathbb{C} .
$$

For generic $a$ the family has a fiber of type $I I^{*}$ over $t=\infty$ and two fibers of type $I_{1}$ over the zeros of $\Delta=4+27(t-a)^{2}$. As in Examples 5.8 and 6.2 we associate to $Y_{a}$ a $K 3$ surface with a non-symplectic automorphism with zero-dimensional fixed locus. Let $p: P \rightarrow \mathbb{P}^{1}$ be a non-trivial principal homogeneous space of $\pi_{a}$ given by an element of order 11 in the fiber $\pi_{a}^{-1}(0)$. The surface $Z$, obtained after blowing up the intersection point of the components of multiplicity 5 and 6 in $p^{-1}(\infty)$ and then blowing down the proper transform of the fiber $p^{-1}(\infty)$, is a $\log$ Enriques surface of index 11 with two singular points. By [35, Theorem 5.1] the canonical cover of $Z$ is a $K 3$ surface and a generator of the Galois group of the covering is a non-symplectic order 11 automorphism with two isolated fixed points. 
Theorem 7.3. The fixed locus of a non-symplectic automorphism $\sigma$ of order 11 of a K3 surface is either

- the union of one point of type 1 and one of type 4 if $S(\sigma) \cong U(11)$, or

- the disjoint union of $n_{i}$ isolated points of type $i$, a smooth curve of genus $g$ and $k$ smooth rational curves where $\left(g, k, n_{i}\right)$ appears in a row of Table 4 .

The same table gives the corresponding invariant lattice $S(\sigma)$ and its orthogonal complement $T(\sigma)$.

Proof. The lattices in Table 4 can be found by means of Theorem 1.1 and Theorem 2.1. For any such lattice $S(\sigma)$ the invariants $g, k, n_{i}$ of $X^{\sigma}$ can be computed by means of Lemma 2.3. Theorem 2.4 and Corollary 2.9.

We will now show that a $K 3$ surface $X$ with a non-symplectic automorphism $\sigma$ of order 11 such that $S_{X}=S(\sigma)$ belongs to one of the two families in Examples 7.1, 7.2. This will also show that all cases in Table 4 do appear. Note that also in this case $X$ admits an elliptic fibration, since $S(\sigma)$ contains a copy of either $U$ or $U(11)$.

$S(\sigma) \cong U$ A $K 3$ surface $X$ with $S_{X}=S(\sigma)$ has a jacobian fibration with no reducible fibers. The order 11 automorphism acts on the basis of the fibration with two fixed points i.e. it preserves two fibers. By Theorem 7.3 we know that the fixed locus contains one elliptic curve and two points. Thus there is one singular fiber with two fixed points and a smooth fiber, which is pointwise fixed. Since $\sigma$ acts on the basis of the fibration and $\mathcal{X}(X)=24$, it follows that the $\sigma$-invariant singular fiber is of Kodaira type II. Thus we are in Example 7.1.

$S(\sigma) \cong U \oplus A_{10}$ By Theorem 7.3 the fixed locus contains one smooth rational curve $R$ and 11 points. Note that $X$ in this case has a jacobian fibration with one fiber of type $I_{11}$ and $R$ is necessarily a component of this reducible fiber. Hence the fiber $I_{11}$ contains 9 fixed points. The remaining two fixed points belong to an irreducible fiber of type $I I$. Thus we are in Example 7.1

$S(\sigma) \cong U(11)$ By [26, Corollary 3,§3] a $K 3$ surface $X$ with $S_{X}=S(\sigma)$ carries an elliptic fibration $\pi: X \rightarrow \mathbb{P}^{1}$ (not jacobian). By Theorem 7.3 we know that there are no curves in the fixed locus and two isolated points. Hence $\pi$ has at most one reducible fiber of type $I I$. Since $\sigma$ induces an order 11 automorphism of $\mathbb{P}^{1}$, then there are two fibers which are preserved. These are the fiber of type $I I$ and a smooth elliptic fiber, which we can assume to be over $\infty$ and 0. For the generic $K 3$ the other singular fibers are 22 of type $I_{1}$, divided in two orbits.

The quotient $X /\langle\sigma\rangle$ is a log Enriques surface of index 11 (see Lemma 1.7 and its proof, 25]) with two singular points (the images of the fixed points on $X$ ) and an elliptic fibration $\bar{\pi}: X /\langle\sigma\rangle \rightarrow \mathbb{P}^{1}$. We can now consider the minimal resolution of $X /\langle\sigma\rangle$, where the proper transform of $\bar{\pi}^{-1}(\infty)$ is a $(-1)$-curve. After contracting this $(-1)$-curve we obtain a smooth rational elliptic surface $Y \rightarrow \mathbb{P}^{1}$ with 11 fibers of type $I_{0}$, one fiber of type $I I^{*}$ and two fibers of type $I_{1}$. Thus we are in Example 7.2 . 


\begin{tabular}{c|c|c|c|c|c|c}
$n_{1}=n_{2}$ & $n_{3}$ & $n_{4}$ & $n_{5}=n_{6}$ & $k$ & $T(\sigma)$ & $S(\sigma)$ \\
\hline 3 & 2 & 1 & 0 & 1 & $U \oplus H_{13} \oplus E_{8}$ & $H_{13} \oplus E_{8}$
\end{tabular}

TABle 5. Order 13

\begin{tabular}{c|c|c|c|c|c|c}
$n_{1}=n_{2}=n_{3}=n_{4}$ & $n_{5}=n_{8}$ & $n_{6}$ & $n_{7}$ & $k$ & $T(\sigma)$ & $S(\sigma)$ \\
\hline 0 & 1 & 2 & 3 & 0 & $U \oplus U \oplus E_{8} \oplus L_{17}$ & $U \oplus L_{17}$
\end{tabular}

TABLE 6. Order 17

Example 7.4 ([11]). Let $X$ be the $K 3$ surface with non-symplectic automorphism:

$$
y^{2}=x^{3}+t^{5} x+t^{2}, \sigma(x, y, t)=\left(\zeta_{11}^{5} x, \zeta_{11}^{2} y, \zeta_{11}^{2} t\right) .
$$

The elliptic fibration has one singular fiber of type $I V$, one of type $I I I^{*}$ and 11 of type $I_{1}$. This is the only $K 3$ surface with order 11 automorphism such that $\operatorname{rank} T(\sigma)=10$. Note that the fiber of type $I I I^{*}$ contains 7 fixed points and a rational fixed curve, while the fiber of type $I V$ contains 4 fixed points.

\section{ORDER $13,17,19$}

$K 3$ surfaces with non-symplectic automorphisms of order 13,17 and 19 are well known and studied in [11] and 23. The following examples are due to Kondō.

Example 8.1. Let $X$ be the $K 3$ surface with non-symplectic automorphism:

$$
y^{2}=x^{3}+t^{5} x+t, \quad \sigma(x, y, t)=\left(\zeta_{13}^{5} x, \zeta_{13} y, \zeta_{13}^{2} t\right) .
$$

The elliptic fibration has one singular fiber of type $I I$, one of type $I I I^{*}$ and 13 fibers of type $I_{1}$. Note that the fiber of type $I I I^{*}$ contains 7 fixed points and a rational fixed curve, while the fiber of type $I I$ contains 2 fixed points.

Example 8.2. Let $X$ be the $K 3$ surface with non-symplectic automorphism:

$$
y^{2}=x^{3}+t^{7} x+t^{2}, \quad \sigma(x, y, t)=\left(\zeta_{17}^{7} x, \zeta_{17}^{2} y, \zeta_{17}^{2} t\right) .
$$

The elliptic fibration has one singular fiber of type $I V$, one of type $I I I$ and 17 fibers of type $I_{1}$. Note that the fiber of type $I V$ contains 4 fixed points, while the fiber of type $I I I$ contains 3 fixed points.

Example 8.3. Let $X$ be the $K 3$ surface with non-symplectic automorphism:

$$
y^{2}=x^{3}+t^{7} x+t, \quad \sigma(x, y, t)=\left(\zeta_{19}^{7} x, \zeta_{19} y, \zeta_{19}^{2} t\right) .
$$

The elliptic fibration has one singular fiber of type $I I$, one of type $I I I$ and 19 fibers of type $I_{1}$. Note that the fiber of type $I I$ contains 2 fixed points, while the fiber of type $I I I$ contains 3 fixed points.

Theorem 8.4. A K3 surface with a non-symplectic automorphism $\sigma$ of order 13,17 or 19 is isomorphic to the surface in Example 8.1, 8.2 or 8.3 respectively.

The fixed locus of such automorphism is the union of $n_{i}$ points of type $i$ and $k$ smooth rational curves, as described in Tables 5, 6] and 7 respectively. The same table gives the corresponding invariant lattices $S(\sigma)$ and their orthogonal complements. 


\begin{tabular}{c|c|c|c|c|c}
$n_{1}=n_{2}=n_{3}=n_{8}=n_{9}$ & $n_{4}=n_{6}=n_{7}$ & $n_{5}$ & $k$ & $T(\sigma)$ & $S(\sigma)$ \\
\hline 0 & 1 & 2 & 0 & $K_{19}(-1) \oplus E_{8} \oplus E_{8}$ & $U \oplus K_{19}$
\end{tabular}

TABle 7. Order 19

Proof. The lattices in tables 5,6 and 7 can be found by means of Theorem 1.1 and Theorem 2.1, For any such lattice $S(\sigma)$ the invariants $g, k, n_{i}$ of the fixed locus $X^{\sigma}$ can be computed by means of Lemma 2.3. Theorem 2.4 and Corollary 2.9]

We now show that a K3 surface with a non-symplectic automorphism of order 13,17 or 19 is as in Example 8.1, 8.2 or 8.3 respectively.

If $p=13$ then $S(\sigma) \cong H_{13} \oplus E_{8}$. Let $e_{1}, e_{2}$ be the generators of $H_{13}$ with intersection matrix as in section 1 and $f \in E_{8}$. The vectors $e_{1}-e_{2}+f, e_{2}+f$ and a basis of $f^{\perp} \cong E_{7} \subset E_{8}$ generate a primitive sublattice $S$ of $S(\sigma)$ isometric to $U \oplus E_{7}$ such that $S^{\perp}$ contains no $(-2)$-curves. It follows that $X$ admits a jacobian elliptic fibration $\pi$ with a unique reducible fiber $F$ of type $I I I^{*}$. Observe that $\sigma$ induces a non-trivially action on the basis of $\pi$, since otherwise the general fiber would have an order 13 automorphism with a fixed point (the intersection with a section of $\pi$ ). Thus $\sigma$ preserves exactly two fibers of $\pi$. Since $\mathcal{X}(X)=24$ and $\mathcal{X}(F)=9$, then $\pi$ has also a $\sigma$-orbit of 13 singular fibers of type $I_{1}$ and a $\sigma$-invariant fiber of type $I I$. Working as in the proof of Theorem 6.3 it can be proved that there is only one jacobian fibration with this property (see also [23, §4]). Thus $X$ is isomorphic to the surface in Example 8.1.

The proofs for $p=17,19$ are similar. In these cases $S(\sigma)$ contains a primitive sublattice $S \cong U \oplus A_{2} \oplus A_{1}$ and $S \cong U \oplus A_{1}$ respectively such that $S^{\perp}$ contains no $(-2)$-curves. Thus the surface admits a jacobian fibration with reducible fibers of types $\tilde{A}_{2} \oplus \tilde{A}_{1}$ and $\tilde{A}_{1}$ respectively. This implies, together with the fact that $\sigma$ acts non-trivially on the basis of the fibration and a computation of $\mathcal{X}(X)$, that $X$ is isomorphic to either the surface in Example 8.2 or 8.3

\section{Moduli spaces}

Let $\rho \in \mathrm{O}\left(L_{K 3}\right)$ be an isometry of prime order $p$ with hyperbolic invariant lattice

$$
S(\rho)=\left\{x \in L_{K 3}: \rho(x)=x\right\}
$$

and let $[\rho]$ be its conjugacy class in $\mathrm{O}\left(L_{K 3}\right)$. A $[\rho]$-polarized $K 3$ surface is a pair $(X, \sigma)$ where $X$ is a $K 3$ surface and $\sigma$ a non-symplectic automorphism of $X$ of order $p$ such that

$$
\sigma^{*}\left(\omega_{X}\right)=\zeta_{p} \omega_{X} \quad \text { and } \quad \sigma^{*}=\phi \circ \rho \circ \phi^{-1}
$$

for some isometry $\phi: L_{K 3} \rightarrow H^{2}(X, \mathbb{Z})$, which is called a marking. Two $[\rho]-$ polarized $K 3$ surfaces $(X, \sigma),\left(X^{\prime}, \sigma^{\prime}\right)$ are isomorphic if there exists an isomorphism $f: X \rightarrow X^{\prime}$ such that $f^{-1} \circ \sigma^{\prime} \circ f=\sigma$.

Observe that a $[\rho]$-polarized $K 3$ surface is algebraic by [18, Theorem 3.1]. If $h$ is an ample class in $S_{X}$, then the average $\sum_{i=1}^{p}\left(\sigma^{*}\right)^{i}(h)$ is an ample class in $S\left(\sigma^{*}\right)$. This implies that any marked $[\rho]$-polarized $K 3$ surface is $S(\rho)$-ample polarized (see [8, $\S 10])$

A moduli space for such polarized surfaces can be constructed as follows (see 8 , $\S 11])$. Let $S(\rho)^{\perp}=T(\rho)$ and $V^{\rho}=\left\{x \in L_{K 3} \otimes \mathbb{C}: \rho_{\mathbb{C}}(x)=\zeta_{p} x\right\} \subset T(\rho) \otimes \mathbb{C}$ be 
an eigenspace of the natural extension of $\rho$ to $L_{K 3} \otimes \mathbb{C}$. Consider the space

$$
D^{\rho}=\left\{w \in \mathbb{P}\left(V^{\rho}\right):(w, \bar{w})>0,(w, w)=0\right\} .
$$

This is a type IV Hermitian symmetric space of dimension $r(T(\rho))-2$ if $p=2$ and it is isomorphic to a complex ball of dimension $r(T(\rho)) /(p-1)-1$ if $p>2$ (note that if $\zeta_{p} \notin \mathbb{R}$ then the condition $(w, w)=0$ is automatically true).

Furthermore, consider the divisor

$$
\Delta^{\rho}=\bigcup_{\delta \in T(\rho), \delta^{2}=-2} D^{\rho} \cap \delta^{\perp}
$$

and the discrete group $\Gamma^{\rho}=\left\{\gamma \in \mathrm{O}\left(L_{K 3}\right): \gamma \circ \rho=\rho \circ \gamma\right\}$.

Theorem 9.1. The orbit space $\mathcal{M}^{\rho}:=\Gamma^{\rho} \backslash\left(D^{\rho} \backslash \Delta^{\rho}\right)$ parametrizes isomorphism classes of $[\rho]$-polarized $K 3$ surfaces.

Proof. Let $(X, \sigma)$ be a $[\rho]$-polarized $K 3$ surface and $\phi: L_{K 3} \rightarrow H^{2}(X, \mathbb{Z})$ be an isometry such that $\sigma^{*}=\phi \circ \rho \circ \phi^{-1}$. Since $\sigma^{*}\left(\omega_{X}\right)=\zeta_{p} \omega_{X}$, then $\ell:=\phi_{\mathbb{C}}^{-1}\left(\mathbb{C} \omega_{X}\right) \in$ $D^{\rho}$. If $\ell \in \delta^{\perp}$ for some $\delta \in T(\rho), \delta^{2}=-2$, then either $\phi(\delta)$ or $\phi(-\delta)$ would be an effective class $x$ such that $\sigma^{*}(x)+\cdots+\left(\sigma^{*}\right)^{p-1}(x)=-x$. This gives a contradiction since the left side is an effective divisor and the right side is not, thus $\ell \notin \Delta^{\rho}$. An isometry $\phi^{\prime}$ also satisfies $\sigma^{*}=\phi^{\prime} \circ \rho \circ \phi^{\prime-1}$ if and only if $\phi^{-1} \circ \phi^{\prime} \in \Gamma^{\rho}$. It is easy to check that two isomorphic $[\rho]$-polarized $K 3$ surfaces give the same point $\ell \in D^{\rho}$ modulo $\Gamma^{\rho}$.

Conversely, let $\ell \in D^{\rho} \backslash \Delta^{\rho}$. By the surjectivity theorem of the period map [5, $\S \mathrm{X}$ ] and [8, Theorem 10.1] there exist a $K 3$ surface and a marking $\phi: L_{K 3} \rightarrow H^{2}(X, \mathbb{Z})$ such that $\phi_{\mathbb{C}}(\ell)=\mathbb{C} \omega_{X}$ and $\phi_{\mid S(\rho)}: S(\rho) \rightarrow S_{X}$ is an ample polarization (see 8, $\S 10])$. Let $\psi=\phi \circ \rho \circ \phi^{-1}$, then $\psi_{\mathbb{C}}(\ell)=\ell$ and its invariant lattice $S(\psi)=\phi(S(\rho))$ contains an ample class. Moreover, $S(\psi)^{\perp} \cap \mathbb{C} \omega_{X}^{\perp}$ contains no elements of selfintersection -2 since $\ell \notin \Delta^{\rho}$. Thus, by the global Torelli Theorem [5, §IX] there is a unique automorphism $\sigma$ of $X$ such that $\sigma^{*}=\psi$. It is clear that $\sigma$ is non-symplectic of order $p$ and that $\sigma^{*}\left(\omega_{X}\right)=\zeta_{p} \omega_{X}$.

Remark 9.2. In [8, Theorem 11.3] it is proved that $\Gamma_{0}^{\rho} \backslash\left(D^{\rho} \backslash \Delta^{\rho}\right)$, where $\Gamma_{0}^{\rho}=\{\gamma \in$ $\left.\Gamma^{\rho}: \gamma_{\mid S(\rho)}=i d\right\}$, parametrizes isomorphism classes of $[\rho]$-polarized $K 3$ surfaces with the extra data of an ample polarization $j: S(\rho) \rightarrow S_{X}$ (see also [8, Remark 11.4]).

The following result says when two $K 3$ surfaces with non-symplectic automorphisms of order $p$ belong to the same moduli space $\mathcal{M}^{\rho}$.

Proposition 9.3. Two pairs $\left(X_{1}, \sigma_{1}\right),\left(X_{2}, \sigma_{2}\right)$ of $K 3$ surfaces with non-symplectic automorphisms of order $p$ are polarized by the same $\rho \in O\left(L_{K_{3}}\right)$ if and only if $S\left(\sigma_{1}\right) \cong S\left(\sigma_{2}\right)$. This is also equivalent to say that $X_{1}^{\sigma_{1}}$ is homeomorphic to $X_{2}^{\sigma_{2}}$ for $p>2$.

Proof. It is clear that two $[\rho]$-polarized $K 3$ surfaces have $S\left(\sigma_{1}\right) \cong S(\rho) \cong S\left(\sigma_{2}\right)$. In sections $\S 5,6,7,8$ we proved that a $K 3$ surface with a non-symplectic automorphism $\sigma$ of order $p=5,7,11,13,17,19$ and given invariant lattice $S(\sigma)=S_{X}$ belongs to an irreducible family. A similar result holds for $p=2,3$ by [19, §4] and [2, §5]. This implies that two pairs with $S\left(\sigma_{1}\right) \cong S\left(\sigma_{2}\right)$ belong to the same irreducible component $\mathcal{M}^{\rho}$. The last statement follows from Remark 2.10,

Remark 9.4. In [18, V.V. Nikulin proved that the action of a symplectic automorphism on the $K 3$ lattice (up to conjugacy) only depends on the number of its fixed 
points. Proposition 9.3 gives a similar statement for non-symplectic automorphisms of prime order $p>2$.

By Theorem 9.1 the moduli space of $[\rho]$-polarized $K 3$ surfaces is an irreducible quasi-projective variety. Moreover, as proved in [8. Theorem 11.7], any point $\ell \in D^{\rho}$ is the period point of some $\left[\rho^{\prime}\right]$-polarized $K 3$ surface, where $\rho^{\prime}$ has order $p$ and $\left[\rho^{\prime}\right]$ is not equal to $[\rho]$ if $\ell \in \Delta^{\rho}$. Thus, the quotient $\Gamma^{\rho} \backslash D^{\rho}$ is an irreducible subvariety of the moduli space $\mathcal{M}_{K 3}^{p}$ of $K 3$ surfaces with a non-symplectic automorphism of order $p$. We are interested in identifying the maximal irreducible subvarieties of this type in $\mathcal{M}_{K 3}^{p}$, or equivalently, its irreducible components.

Theorem 9.5. The following table gives the number \# of irreducible components of the moduli space $\mathcal{M}_{K 3}^{p}$, their dimensions and the Picard lattice $S(\sigma)$ of the generic $K 3$ surface in each component, for any prime $p$.

\begin{tabular}{cccc}
$p$ & $\#$ & $\operatorname{dim}$ & $S(\sigma)$ \\
\hline 2 & 2 & 19,18 & $(2), U(2)$ \\
3 & 3 & $9,9,6$ & $U, U(3), U(3) \oplus E_{6}^{*}(3)$ \\
5 & 2 & 4,3 & $H_{5}, H_{5} \oplus A_{4}^{*}(5)$ \\
7 & 2 & 2,2 & $U \oplus K_{7}, U(7) \oplus K_{7}$ \\
11 & 2 & 1,1 & $U, U(11)$ \\
13 & 1 & 0 & $H_{13} \oplus E_{8}$ \\
17 & 1 & 0 & $U \oplus L_{17}$ \\
19 & 1 & 0 & $U \oplus K_{19}$
\end{tabular}

TABLE 8. Irreducible components of $\mathcal{M}_{K 3}^{p}$

Proof. Observe that the moduli space $\mathcal{M}^{\rho}$ is in the closure of $\mathcal{M}^{\rho_{2}}$ if and only if there is $\rho_{1} \in[\rho]$ such that $D^{\rho_{1}} \subset D^{\rho_{2}}$, i.e. $V^{\rho_{1}} \subset V^{\rho_{2}}$. This is equivalent to say that $T\left(\rho_{1}\right) \subset T\left(\rho_{2}\right)$ and $\rho_{2}=\rho_{1}$ on $T\left(\rho_{1}\right)$.

If $p=2$, then $\rho_{i}=-i d$ on $T\left(\rho_{i}\right)$, hence $\mathcal{M}^{\rho} \subset \mathcal{M}^{\rho_{2}}$ if and only if $T\left(\rho_{1}\right) \subset T\left(\rho_{2}\right)$, or equivalently $S\left(\rho_{1}\right) \supset S\left(\rho_{2}\right)$. As a consequence of Theorem 3.1, the invariant lattice $S(\sigma)$ of a non-symplectic involution contains a primitive sublattice which is isometric to either (2) or $U(2)$. This implies, since (2) clearly is not a sublattice of $U(2)$, that $\mathcal{M}_{K 3}^{2}$ has two irreducible components whose generic elements are K3 surfaces with $S_{X}$ isomorphic to (2) and $U(2)$ respectively.

The case $p=3$ is [2, Theorem 5.6]. In Theorem [5.3 we proved that a $K 3$ surface with an order 5 non-symplectic automorphism either belongs to the family in Example 5.1 or to the one in Example 5.2. These two families are irreducible and of dimensions 4 and 3 respectively. Thus we only need to prove that the second component is not contained in the first one. Assume that the generic pair $\left(X, \sigma_{1}\right)$ in the first family (we assume $\sigma_{1}^{*}\left(\omega_{X}\right)=\zeta_{5} \omega_{X}$ ), with $S_{X}=S\left(\sigma_{1}\right) \cong H_{5} \oplus A_{4}^{*}(5)$, also belongs to the second family. Since the orthogonal complement of $H_{5}$ in $S_{X}$ contains no $(-2)$-curves, then by Theorem 9.1, $X$ carries an automorphism $\sigma_{2}$ of order 5 such that $\sigma_{2}^{*}\left(\omega_{X}\right)=\zeta_{5} \omega_{X}$ and $S\left(\sigma_{2}\right) \cong H_{5}$. If $h \in S\left(\sigma_{1}\right), h^{2}=2$ is as in $\S 5$, then the associated morphism is a double cover of $\mathbb{P}^{2}$ branched along a smooth sextic curve $C$ (since $A_{4}^{*}(5)$ contains no (-2)-curves). Since $h$ is fixed by $\sigma_{i}^{*}, i=1,2$, then $\sigma_{i}$ induces a projectivity $\bar{\sigma}_{i}$ of $\mathbb{P}^{2}$ which preserves $C$. The automorphism group 
of $C$ is finite, thus $\bar{\sigma}_{1}^{4} \circ \bar{\sigma}_{2}$ has finite order and $\sigma_{1}^{4} \circ \sigma_{2}$ is a symplectic automorphism of finite order of $X$. By [18] this would imply that the Picard lattice of $X$ has rank $>8$, giving a contradiction.

If $p=7$, then in Theorem 6.3 we proved that a $K 3$ surface with an order 7 non-symplectic automorphism either belongs to the family in Example 6.1 or to the one in Example 6.2. Both are clearly irreducible of dimension 2, thus they are the irreducible components of $\mathcal{M}_{K 3}^{7}$.

If $p=11$, then in Theorem 7.3 we proved that a $K 3$ surface with an order 11 non-symplectic automorphism either belongs to the family in Example 7.1 or to the one in Example 7.2. Both are clearly irreducible and 1-dimensional, thus they are the irreducible components of $\mathcal{M}_{K 3}^{11}$.

If $p=13,17$ or 19 then by Theorem 8.4 the moduli space $\mathcal{M}_{K 3}^{p}$ is irreducible and 0-dimensional.

Remark 9.6. The general members of the two irreducible components of $\mathcal{M}_{K 3}^{2}$ are double covers of the plane branched along a smooth sextic curve (if $S_{X} \cong(2)$ ) and double covers of a quadric branched along a smooth curve of bidegree $(4,4)$ (if $S_{X} \cong U(2)$ ). Projective models for the general members of $\mathcal{M}_{K 3}^{3}$ are described in [2] and [31].

\section{REFERENCES}

[1] V. Alexeev, V.V. Nikulin. Del Pezzo and K3 surfaces. MSJ Memoirs, Vol. 15, Mathematical Society of Japan, Tokyo, 2006.

[2] M. Artebani, A. Sarti. Non symplectic automorphisms of order 3 on $K 3$ surfaces. Math. Ann. (2008), 342, 903-921.

[3] M.F. Atiyah, I.M. Singer. The index of elliptic operators: III. Ann. of Math., 87 (1968), 546-604.

[4] W. Barth, C. Peters, A. van de Ven. Compact complex surfaces. Springer, Berlin, Heidelberg, New York, 1984.

[5] A. Beauville, J.-P. Bourguignon, M. Demazure. Géometrie des surfaces K3: modules et périodes. Astérisque, vol. 126, Soc. Math. France, 1985.

[6] G.E. Bredon. Introduction to compact transformation groups. Pure and Applied Math. 46, Academic Press, New York-London, 1972.

[7] F. Cossec, I. Dolgachev. Enriques Surfaces I. Progress in Mathematics, 76. Birkhäuser Boston, Inc., Boston, MA, 1989.

[8] I. Dolgachev, S. Kondō. Moduli spaces of $K 3$ surfaces and complex ball quotients. Arithmetic and geometry around hypergeometric functions, 43-100, Progr. Math., 260, Birkhäuser, Basel, 2007.

[9] M. J. Greenberg. Lectures on algebraic topology. W. A. Benjamin, Inc., New YorkAmsterdam, 1967.

[10] V.M. Kharlamov. The topological type of nonsingular surfaces in $\mathbb{P}^{3} \mathbb{R}$ of degree four. Funct. Anal. Appl.,10:4 (1976) 295-304.

[11] S. Kondō. Automorphisms of algebraic $K 3$ surfaces which act trivially on Picard groups. J. Math. Soc. Japan, 44 (1992), 75-98.

[12] S. Kondō. The moduli space of 5 points on $\mathbb{P}^{1}$ and $K 3$ surfaces. Progress in Mathematics, 260 (2007), 189-206.

[13] S. Kondō. The moduli space of curves of genus 4 and Deligne-Mostow's complex reflection groups. Advanced Studies in Pure Math., 36, Algebraic Geometry 2000, Azumino, 383-400.

[14] N. Machida, K. Oguiso. On $K 3$ surfaces admitting finite non-symplectic group actions. J. Math. Sci. Univ. Tokyo, 5, n.2 (1998), 273-297.

[15] J. Milnor. On simply connected 4-manifolds. International symposium in algebraic topology, (1958), Universidad Nacional Autonoma de México and UNESCO, Mexico city, 122-128.

[16] R. Miranda. The basic theory of elliptic surfaces. Dottorato di ricerca in Matematica, ETS Editrice, Pisa (1989). 
[17] Y. Namikawa. Periods of Eriques surfaces. Math. Ann. 270 (1985), 201-222.

[18] V.V. Nikulin. Finite groups of automorphisms of Kählerian surfaces of type $K 3$. Moscow Math. Soc., 38 (1980), 71-137.

[19] V.V. Nikulin. Integral symmetric bilinear forms and some of their applications. Math. USSR Izv., 14 (1980), 103-167.

[20] V.V. Nikulin. Factor groups of groups of the automorphisms of hyperbolic forms with respect to subgroups generated by 2-reflections. Soviet Math. Dokl., 20 (1979), 1156-1158.

[21] V.V. Nikulin. Discrete reflection groups in Lobachevsky spaces and algebraic surfaces. Proceedings of the International Congress of Mathematicians, Vol. 1, 2 (Berkeley, Calif., 1986), 654-671, Amer. Math. Soc., Providence, RI, 1987.

[22] K. Oguiso, D-Q. Zhang. K3 surfaces with order 11 automorphisms. arXiv:math/9907020v1

[23] K. Oguiso, D-Q. Zhang. On Vorontsov's theorem on $K 3$ surfaces with non-symplectic group actions. Proc. Amer. Math. Soc. 128 (2000), no. 6, 1571-1580.

[24] K. Oguiso, D-Q. Zhang. K3 surfaces with order five automorphisms. J. Math. Kyoto Univ. (1998) 419-438.

[25] K. Oguiso, D-Q. Zhang. On extremal log Enriques surfaces, II. Tohoku Math. J. 50 (1998), 419-436.

[26] I.I. Pjateckii-Shapiro, I.R. Shafarevich. A Torelli Theorem for algebraic surfaces of type K3. Izv. Akad. Nauk SSSR Ser. Mat. 35 (1971) 530-572. Math. USSR Izvestija Vol. 5 (1971) $547-588$.

[27] A.N. Rudakov, I. Shafarevich. Surfaces of type $K 3$ over fields of finite characteristic. In: I. Shafarevich, Collected mathematical papers, Springer, Berlin (1989), 657-714.

[28] J.-P. Serre. A course in arithmetic. Graduate Texts in Mathematics, No.7, Springer-Verlag, New York-Heidelberg, 1973.

[29] T. Shioda. An explicit algorithm for computing the Picard number of certain algebraic $K 3$ surfaces. American Journal of Mathematics, 108 (1986), 415-432.

[30] N.J.A. Sloane, AT\&T Labs-Research and G. Nebe. Catalogue of Lattices. University of Ulm, http://www.research.att.com/ njas/lattices.

[31] S. Taki. Classification of non-symplectic automorphisms of order 3 on $K 3$ surfaces. To appear in Math. Nachr. (2008).

[32] S.P. Vorontsov. Automorphisms of even lattices arising in connection with automorphisms of algebraic K3-surfaces. Vestnik Moskov. Univ. Ser. I Mat. Mekh. (1983), no. 2, 19-21.

[33] D.-Q. Zhang. Quotients of K3 surfaces modulo involutions. Japan. J. Math. (N.S.) 24 (1998), no. $2,335-366$.

[34] D.-Q. Zhang. Normal Algebraic Surfaces with trivial tricanonical divisors. Publ. RIMS, Kyoto Univ. 33 (1997), 427-442.

[35] D.-Q. Zhang. Logarithmic Enriques surfaces. J. Math. Kyoto Univ. 31-2 (1991), 419-466.

[36] D.-Q. Zhang. Normal Logarithmic Enriques surfaces, II. J. Math. Kyoto Univ. 33-2 (1993), 357-397.

Michela Artebani, Departamento de Matemática, Universidad de Concepción, Casilla 160C, Concepción, Chile. e-mail: martebani@udec.cl.

Alessandra Sarti, Université de Poitiers, Laboratoire de Mathématiques et Applications, Téléport 2 Boulevard Marie et Pierre Curie BP 30179, 86962 Futuroscope Chasseneuil Cedex, France. e-mail sarti@math.univ-poitiers.fr,

URL http://www. mathematik.uni-mainz.de/ sarti.

Shingo Taki, Graduate School of Mathematics, Nagoya University, Chikusa-ku Nagoya 464-8602 Japan. e-mail m04022x@math.nagoya-u.ac.jp. 


\title{
Appendix: On Naruki's $K 3$ surface
}

\author{
Shigeyuki Kondđ1
}

As a moduli space of some $K 3$ surfaces with a non-symplectic automorphism of order 5, the quintic del Pezzo surface appears ( $\mathrm{K} 2$, see the following Remark 1.5 for more details). In this appendix we shall give a similar example in case of $K 3$ surfaces with a non-symplectic automorphism of order 7 .

1.1. Naruki's $K 3$ surface. Let $\zeta=e^{2 \pi \sqrt{-1} / 7}$. We introduce a hermitian form of signature $(1,2)$ with variables $z=\left(z_{1}, z_{2}, z_{3}\right)$ by setting

$$
H(z)=(\zeta+\bar{\zeta}) z_{1} \bar{z}_{1}-z_{2} \bar{z}_{2}-z_{3} \bar{z}_{3} .
$$

We denote by $S U(1,2)$ the group of $(3,3)$-matrices of determinant 1 which are unitary with respect to $H$. The group $S U(1,2)$ naturally acts on the complex ball of dimension 2

$$
D=\left\{\left(z_{1}, z_{2}, z_{3}\right) \in \mathbb{P}^{2}: H(z)>0\right\} .
$$

We denote by $\Gamma$ the subgroup of $S U(1,2)$ consisting of elements whose entries are integers in $\mathbb{Q}(\zeta)$. It is known that $\Gamma$ acts on $D$ properly discontinuously and the quotient $D / \Gamma$ is compact. We further denote by $\Gamma^{\prime}$ the subgroup of $\Gamma$ consisting of matrices which are congruent to the identity matrix modulo the principal ideal $P$ generated by $1-\zeta$. Naruki $[\mathrm{N}]$ showed that the quotient $D / \Gamma^{\prime}$ is isomorphic to a $K 3$ surface $X$.

1.2. $K 3$ surfaces with a non-symplectic automorphism of order 7 . In the following we shall show that the Naruki's $K 3$ surface $X$ is the moduli space of pairs of a $K 3$ surface and a non-symplectic automorphism of order 7 .

Let $S=U(7) \oplus K_{7}$ and its orthogonal complement $T=U \oplus U(7) \oplus E_{8} \oplus A_{6}$ in $L_{K 3}$ (see Table 3 ).

Remark-Definition 1.1. By [RS, Theorem 2.1] it follows that $T$ is isomorphic to $T^{\prime}=U \oplus U \oplus K_{7} \oplus A_{6}^{2}$. An order 7 isometry without non-zero fixed vectors on $T$ can be thus explicitely described as follows.

Let $U_{1}, U_{2}$ be two copies of the hyperbolic plane $U$ and let $e_{i}, f_{i}$ be a basis of $U_{i}$, $(i=1,2)$ satisfying $e_{i}^{2}=f_{i}^{2}=0,\left(e_{i}, f_{i}\right)=1$. Let $x, y$ be a basis of $K_{7}$ satisfying $x^{2}=-2, y^{2}=-4,(x, y)=1$. Let $\rho_{0}$ be the isometry of $U_{1} \oplus U_{2} \oplus K_{7}$ defined by

$$
\begin{array}{ll}
\rho_{0}\left(e_{1}\right)=e_{1}+f_{1}+e_{2}+f_{2}-y & \rho_{0}\left(f_{1}\right)=2 e_{1}+e_{2}+2 f_{2}-y \\
\rho_{0}\left(e_{2}\right)=-f_{1}+e_{2}+f_{2}+x & \rho_{0}\left(f_{2}\right)=-f_{1}+e_{2} \\
\rho_{0}(x)=e_{1}+2 f_{1}-e_{2}+f_{2}-x-y, & \rho_{0}(y)=3 e_{1}-f_{1}+4 e_{2}+3 f_{2}+x-2 y
\end{array}
$$

It is easy to see that $\rho_{0}$ has order 7 and acts trivially on the discriminant group of $U_{1} \oplus U_{2} \oplus K_{7}$.

An easy calculation shows that

$$
v=\left(-1+\zeta^{2}+\zeta^{4}-\zeta^{5}\right) e_{1}+\left(\zeta^{3}-1\right) f_{1}+\left(\zeta-\zeta^{5}\right) e_{2}+\left(\zeta^{2}-\zeta^{5}\right) f_{2}+x+\left(1+\zeta^{5}\right) y
$$

is an eigenvector of $\rho_{0}$ with the eigenvalue $\zeta$ and

$$
(v, \bar{v})=7\left(\zeta+\zeta^{6}\right)
$$

\footnotetext{
${ }^{1}$ Research of the author is partially supported by Grant-in-Aid for Scientific Research A18204001 and Houga-20654001, Japan
} 
On the other hand, let $r_{1}, \ldots, r_{6}$ be a basis of $A_{6}$ such that $r_{i}^{2}=-2,\left(r_{i}, r_{i+1}\right)=1$ and the other $r_{i}$ 's and $r_{j}$ 's are orthogonal. Consider the isometry of $A_{6}$ defined by

$$
\rho_{6}\left(r_{i}\right)=r_{i+1}, \quad(1 \leq i \leq 5), \quad \rho_{6}\left(r_{6}\right)=-\left(r_{1}+r_{2}+r_{3}+r_{4}+r_{5}+r_{6}\right) .
$$

It is easy to see that $\rho_{6}$ acts trivially on the discriminant group of $A_{6}$ and that

$$
w=r_{1}+\left(\zeta^{6}+1\right) r_{2}+\left(1+\zeta^{5}+\zeta^{6}\right) r_{3}-\left(\zeta+\zeta^{2}+\zeta^{3}\right) r_{4}-\left(\zeta+\zeta^{2}\right) r_{5}-\zeta r_{6}
$$

is an eigenvector of $\rho_{6}$ with the eigenvalue $\zeta$ and

$$
(w, \bar{w})=-7 .
$$

Combining $\rho_{0}$ and $\rho_{6}$, we define an isometry $\rho$ of $T$ of order 7 and without nonzero fixed vectors. Moreover the action of $\rho$ on the discriminant group $T^{*} / T$ is trivial.

In Definition-Remark 1.1 we explicitely described an order 7 isometry $\rho$ on $T$ without nonzero fixed vectors and acting trivially on the discriminant group. Hence $\rho$ can be extended to an isometry $\rho$ (we use the same symbol) of $L_{K 3}$ acting trivially on $S$.

Now we consider a $K 3$ surface $Y$ with $S_{Y} \cong S$. Then the transcendental lattice $T_{Y}$ of $Y$ is isomorphic to $T$. We identify $L_{K 3}$ and $H^{2}(Y, \mathbb{Z})$ so that $S=S_{Y}$ and $T=T_{Y}$. If the period $\omega_{Y} \in T \otimes \mathbb{C}$ is an eigenvector of $\rho$, then it follows from the Torelli type theorem for $K 3$ surfaces that $\rho$ can be realized as an automorphism $g$ of $Y$ of order 7: $g^{*}=\rho$. Now consider the eigenspace decomposition of $\rho$ :

$$
T \otimes \mathbb{C}=\oplus_{i=1}^{6} V_{\zeta^{i}}
$$

where $V_{\zeta^{i}}$ is the eigenspace corresponding to the eigenvalue $\zeta^{i}$. The period domain of the pair $(Y, g)$ is given by

$$
D^{\prime}=\left\{\omega \in \mathbb{P}\left(V_{\zeta}\right):\langle\omega, \bar{\omega}\rangle>0\right\} .
$$

Then the above calculations show that the hermitian form on $V_{\zeta}$ defined by $\langle\omega, \bar{\omega}\rangle$ is given by $7 H(\xi)$. We define an arithmetic subgroup $\tilde{\Gamma}$ by

$$
\tilde{\Gamma}=\{\varphi \in \mathrm{O}(T): \varphi \circ \rho=\rho \circ \varphi\}
$$

and a subgroup $\tilde{\Gamma^{\prime}}$ by

$$
\tilde{\Gamma}^{\prime}=\tilde{\Gamma} \cap \operatorname{Ker}\left\{\mathrm{O}(T) \rightarrow \mathrm{O}\left(q_{T}\right)\right\} .
$$

Let $\Delta=\bigcup \delta^{\perp} \cap D^{\prime}$ where $\delta$ moves over all $(-2)$-vectors in $T$. Then $\left(D^{\prime} \backslash \Delta\right) / \tilde{\Gamma^{\prime}}$ is the moduli of the pair $(Y, g)$. Note that $\rho$ has discriminant -1 and is contained in $\tilde{\Gamma^{\prime}}$. Moreover $\rho$ acts trivially on $B$. By using the same method as in [K1], we have

1.3. Theorem. $X \cong D / \Gamma^{\prime} \cong D^{\prime} / \tilde{\Gamma}^{\prime}$.

1.4. Remark. Naruki $[\mathrm{N}]$ showed that $X$ has an elliptic fibration with three singular fibers of type $I_{7}$ in the sense of Kodaira and with 7 sections. Thus $X$ contains 28 smooth rational curves. In particular $X$ has the Picard number 20 . We can see that $\Delta / \Gamma^{\prime}$ consists of 28 curves corresponding to 28 smooth rational curves on $X$. We omit the proof of this fact here. 
1.5. Remark. Let $Z$ be a $K 3$ surface with Picard lattice

$$
S_{Z} \cong\left(\begin{array}{cc}
2 & 1 \\
1 & -2
\end{array}\right) \oplus A_{4} \oplus A_{4}
$$

and with a non-symplectic automorphism $\sigma$ of order 5 acting trivially on $S_{Z}$. The author $\left[\mathrm{K} 2\right.$ showed that the moduli space of ordered 5-points on $\mathbb{P}^{1}$ is isomorphic to the moduli space of the pairs of such $(Z, \sigma)$. Moreover these moduli spaces can be written birationally as an arithmetic quotient of a 2-dimensional complex ball which is isomorphic to the quintic del Pezzo surface.

\section{REFERENCES}

[K1] S. Kondō, A complex hyperbolic structure of the moduli space of curves of genus three, J. reine angew. Math., 525 (2000), 219-232.

[K2] S. Kondō, The moduli space of 5 points on $\mathbb{P}^{1}$ and $K 3$ surfaces, Progress in Mathematics, vol. 260 (2007), 189-206.

[N] I. Naruki, On a K3 surface which is a ball quotient, Max Planck Institute Preprint Series.

[RS] A.N. Rudakov and I. Shafarevich, Surfaces of type K3 over fields of finite characteristic, I. Shafarevich, Collected mathematical papers, Springer, Berlin (1989), 657-714.

Shigeyuki Kondō, Graduate School of Mathematics, Nagoya University, Nagoya, 464-8602, Japan. e-mail kondo@math.nagoya-u.ac.jp 